\title{
Quantum limit of the laser line width in chaotic cavities and statistics of residues of scattering matrix poles
}

\author{
H. Schomerus ${ }^{\text {a }}$, K.M. Frahm ${ }^{\mathrm{b}}$, M. Patra ${ }^{\mathrm{a}}$, C.W.J. Beenakker ${ }^{\mathrm{a}}$ \\ ${ }^{7}$ Instituut Lorentz Unversitert Leiden PO Box 9506 NL 2300 RA Leiden Netherlands \\ ${ }^{\text {b} L a b o r a t o n e ~ d e ~ P h y s t q u e ~ Q u a n t i q u e ~ U M R ~} 5626$ du CNRS Unverstte Paul Sabatret F 31062 \\ Toulouse Ceder 4 Fiance
}

Recerved 1 November 1999

\begin{abstract}
The quantum-limited line width of a lase cavity is enhanced above the Schawlow-Townes value by the Petermann factor $K$, due to the non-oithogonality of the cavity modes We delive the 1elation between the Petermann factor and the residues of poles of the scattering matrix and investigate the statistical properties of the Petermann factor for cavities in which the radiation 1s scattered chaotically For a single scatteing channel wo determine the complete probability distribution of $K$ and find that the average Petcimann factor $\langle K\rangle$ depends non-analytically on the area of the opening, and gieatly exceeds the most probable value For an arbittany number $N$ of scattering channels we calculate $\langle K\rangle$ as a function of the decay rate $\Gamma$ of the lasing mode We find for $N \gg 1$ that for typical values of $\Gamma$ the average Petermann factor $\langle K\rangle \propto \sqrt{N} \gg 1$ is parametıcally la1get than unity (C) 2000 Elsevier Science B V All ıights reserved
\end{abstract}

PACS $4250 \mathrm{Lc}, 4250 \mathrm{Al}, 4260 \mathrm{Da}$

Keywords Petermann factor, Chaotic resonators, Random matix theory

\section{Introduction}

Laser action selects a mode in a cavity and enhances the output intensity in this mode by a non-linea feedback mechanısm Vacuum fluctuations of the electiomagnetıc field ultımately limit the nanowing of the emission spectrum [1] The quantum-limited line

\footnotetext{
* Concsponding author Fax +3171 527-5511

E-mal address henning@lorentz ladenunıvnl (H Schomerus)
} 
width, or Schawlow Townes line width,

$$
\delta \omega_{\mathrm{ST}}=\frac{1}{2} \Gamma^{2} / I
$$

is proportional to the square of the decay rate $\Gamma$ of the lasing cavity mode and inversely proportional to the output power $I$ (in units of photons/s) This is a lower bound for the line width when $\Gamma$ is much less than the line width of the atomic transition and when the lower level of the transition is unoccupied Many years after the work of Schawlow and Townes it was iealized [2-4] that the true fundamental limit is laiger than $\mathrm{Eq}$ ( 11 ) by a factor $K$ that characterizes the non-orthogonality of the cavity modes This excess noise factor, or Petermann factor, has generated an extensive literature [4-10]

Apart from its importance for cavity lasers, the Petermann factor is of fundamental significance in the more general context of scattering theory A lasing cavity mode is associated with a pole of the scattering matıx in the complex fiequency plane We will show that the Petermann factor is proportional to the squared modulus of the 1esidue of this pole Poles of the scattering matrix also determine the position and height of resonances of nucle1, atoms, and molecules [11] Powerful numerical tools that give access to poles even deep in the complex plane have been developed recently [12] They can be used to determine the residues of the poles as well Our work is of relevance for these more general studies, beyond the original application to cavity lasers

Existing theories of the Petermann factor deal with cavities in which the scatteing is essentially one-dimensional, because the geometry has a high degree of symmetiy For such cavities the framework of ray optics provides a simple way to solve the problem in a good approximation [6,7] This approach breaks down if the light propagation in the cavity becomes chaotic, either because of an irregular shape of the boundaries (like for the cavity depicted in Fig 1) or because of randomly placed scatteters The method of random-matrix theory is well-suited for such chaotic cavities $[13,14]$ Instead of considering a single cavity, one studies an ensemble of cavities with small variations in shape and size, or position of the scatterers The distribution of the scattering matrix in this ensemble 1s known Recent work has provided a detalled knowledge on the statıstics of the poles [15-19] Much less is known about the residues [20-22] In this work we fill the remaining gap to a considerable extent

The outline of this paper is as follows In Section 2 we derive the connection between the Petermann factor and the residue of the pole of the lasing mode The residue in turn is seen to be characteristic for the degree of non-orthogonality of the modes In this way we make contact with the existing literature on the Petermann factor $[9,10]$

In Section 3 we study the single-channel case of a scalar scattering matrix This applies to a cavity that is coupled to the outside via a small opening of area $\mathscr{A} \lesssim \lambda^{2} / 2 \pi$ (with $\lambda$ the wavelength of the lasing mode) For preserved time-reversal symmetry (the relevant case in optics) we find that the ensemble average of $K-1$ depends non-analytically $\propto T \ln T^{-1}$ on the transmission probability $T$ through the opening, so that it is beyond the reach of perturbation theory even if $T \ll 1$ We present a 


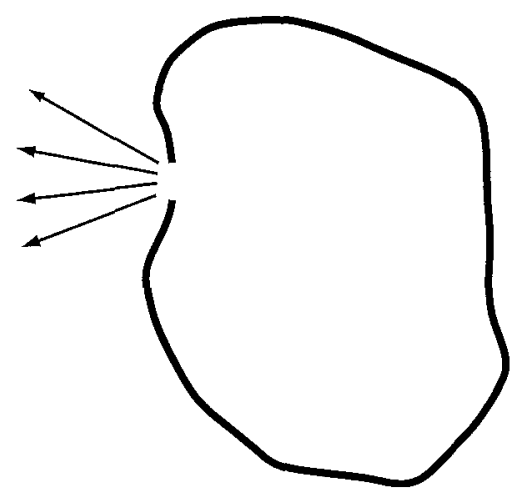

Fig 1 Chaotic cavity that iadiates light from a small opening

complete resummation of the pertubation series that overcomes this obstacle We deilve the conditional distribution $P(K)$ of the Petermann factor at a given decay rate $\Gamma$ of the lasing mode, valid for any value of $T$ The most probable value of $K-1$ is $\propto T$ Hence it is parametrically smaller than the average

In a cavity with such a small opening the deviations of $K$ from unity are very small For larger deviations we study, in Section 4, the mult1-channel case of an $N \times N$ scattering matrix, which corresponds to an opening of area $\mathscr{A} \approx N \lambda^{2} / 2 \pi$ The lasing mode acquiles a decay rate $\Gamma$ of order $\Gamma_{0}=N T A / 2 \pi$ (with $\Delta$ the mean spacing of the cavity modes) We compute the mean Petermann factor as a function of $\Gamma$ for broken time-1eversal symmetry, which is technically simpler than the case of preserved time-reveisal symmetry, but qualitatively simılar We find a parametrically large mean Petermann factor $K \propto \sqrt{N}$

Ou1 conclusions are given in Section 5 The main results of Sections 3 and 4 have been reported in Refs [23,24], 1espectively

\section{Relationship between Petermann factor and residue}

Modes of a closed cavity, in the absence of absorption or amplification, are eigenvalues $\omega_{n}$ of a Hermitian operato1 $H$ This operator can be chosen real if the system possesses time-1eversal symmetry (symmetry index $\beta=1$ ), otherwise it is complex $(\beta=2)$ For a chaotic cavity, $H$ can be modeled by an $M \times M$ Hermitian matrix with independent Gaussian distributed elements

$$
P(H) \propto \exp \left[-\frac{\beta M}{4 \mu^{2}} \mathrm{t} H^{2}\right]
$$

(For $\beta=1$ (2), this is the Gaussian orthogonal (unitary) ensemble [14]) The mean density of eigenvalues is the Wigner semicircle

$$
\rho(\omega)=\frac{M}{2 \pi \mu^{2}} \sqrt{4 \mu^{2}-\omega^{2}}
$$


The mean mode spacing at the center $\omega=0$ is $\Delta=\pi \mu / M$ (The limit $M \rightarrow \infty$ at fixed spacing $\Delta$ of the modes is taken at the end of the calculation )

A small opening in the cavity is described by a 1eal, non-1andom $M \times N$ coupling matrix $W$, with $N$ the number of scatte1ng channels transmitted thiough the opening (For an opening of area $\mathscr{A}, N \simeq 2 \pi \mathscr{A} / \lambda^{2}$ at wavelength $\lambda$ ) Modes of the open cavity are complex eigenvalues (with negative imaginaiy part) of the non-Hermitian matix

$$
\mathscr{H}=H-1 \pi W W^{\top}
$$

In absence of amplification or absoiption, the scattening matıx $S$ at fiequency $\omega$ is related to $\mathscr{H}$ by $[11,25]$

$$
S=\mathbb{1}-2 \pi 1 W^{\dagger}(\omega-\mathscr{P}){ }^{1} W
$$

The scattering matrix is a unitary (and symmetric, for $\beta=1$ ) 1andom $N \times N$ matix, with poles at the eigenvalues of $\mathscr{H}$ It enters the input output relation

$$
a_{m}^{\mathrm{out}}(\omega)=\sum_{n-1}^{N} S_{m n}(\omega) a_{n}^{\mathrm{m}}(\omega),
$$

which ielates the anmihilation operators $a_{m}^{\text {out }}$ of the scatteing states that leave the cavity to the annihilation operators $a_{n}^{\text {in }}$ of states that ente1 the cavity The indices $n, m$ label the scattering channels

We now assume that the cavity is filled with a homogeneous amplifying medium (constant amplification rate $1 / \tau_{a}$ over a large fiequency window $\Omega_{a}=L \Delta, L \gg N$ ) This adds a term $1 / 2 \tau_{a}$ to the eigenvalues, shifting them upwards towards the real axis The scattering matrix

$$
S=\mathbb{1}-2 \pi_{1} W^{\dagger}\left(\omega-\mathscr{H}-1 / 2 \tau_{a}\right){ }^{1} W
$$

is then no longer unitary, and the input-output relation changes to $[26,27]$

$$
a_{m}^{\text {out }}(\omega)=\sum_{n 1}^{N} S_{m n}(\omega) a_{n}^{\text {in }}(\omega)+\sum_{n 1}^{N} Q_{m n}(\omega) b_{n}^{\dagger}(\omega)
$$

All operators fulfill the canonical bosonic commutation relations $\left[a_{n}(\omega), a_{m}^{\dagger}\left(\omega^{\prime}\right)\right]=$ $\delta_{n m} \delta\left(\omega-\omega^{\prime}\right)$ As a consequence,

$$
Q(\omega) Q^{\dagger}(\omega)=S(\omega) S^{\dagger}(\omega)-\mathbb{1}
$$

The operators $b$ descibe the spontaneous emission of photons in the cavity and have expectation value

$$
\left\langle b_{n}^{\dagger}(\omega) b_{m}\left(\omega^{\prime}\right)\right\rangle=\delta_{n m} \delta\left(\omega \quad \omega^{\prime}\right) f(\omega, T),
$$

with $f(\omega, T)=\left[\exp \left(\hbar \omega / k_{B} T\right)-1\right]^{-1}$ the Bose-Einstein distıbution function at frequency $\omega$ and temperature $T$

In the absence of external illumination $\left(\left\langle a^{\dagger+1} a^{m n}\right\rangle=0\right)$, the photon cursent per frequency interval,

$$
I(\omega)=\frac{1}{2 \pi} \sum_{m}^{N}\left\langle a_{m}^{\text {out } \dagger}(\omega) a_{m}^{\text {out }}(\omega)\right\rangle
$$


Is related to the scatteing matrix by Kurchhoff's law [22,23]

$$
I(\omega)=f(\omega, T) \frac{1}{2 \pi} \mathrm{t}_{\mathrm{t}}\left[\mathbb{1}-S^{\dagger}(\omega) S(\omega)\right] .
$$

For $\omega$ near the laser transition we may replace $f$ by the population inversion factor $N_{\text {up }} /\left(N_{\text {low }}-N_{\text {up }}\right)$, where $N_{\text {up }}$ and $N_{\text {low }}$ are the mean occupation numbets of the upper and lower levels of the transition In this way the photon current can be written in the form

$$
I(\omega)=\frac{1}{2 \pi} \frac{N_{\text {up }}}{N_{\text {up }}-N_{\text {low }}} \operatorname{tr}\left[S^{\dagger}(\omega) S(\omega)-\mathbb{1}\right],
$$

that is suttable for an amplifyıng medium. (Alternatıvely, one can associate a negatıve temperature to an amplıfying medium )

The lasing mode is the eigenvalue $\Omega-1 \Gamma / 2$ closest to the real axis, and the laser threshold is reached when the decay rate $\Gamma$ of this mode equals the amplification rate $1 / \tau_{a}$ Near the laser threshold we need to retain only the contribution from the lasing mode (say mode number $l$ ) to the scattering matrix (26),

$$
S_{n m}=-2 \pi 1 \frac{\left(W^{\dagger} U\right)_{n l}\left(U^{-1} W\right)_{l m}}{\omega-\Omega+1 \Gamma / 2-1 / 2 \tau_{a}},
$$

where $U$ is the matrix of right eigenvectors of $\mathscr{H}$ (no summation over $l$ is implied) The photon current near threshold takes the form

$$
I(\omega)=\frac{2 \pi N_{\mathrm{up}}}{N_{\mathrm{up}}-N_{\mathrm{low}}} \frac{\left(U^{\dagger} W W^{\dagger} U\right)_{l l}\left(U^{-1} W W^{\dagger} U^{-1 \dagger}\right)_{l l}}{(\omega-\Omega)^{2}+\frac{1}{4}\left(\Gamma-1 / \tau_{a}\right)^{2}} .
$$

This is a Lorentzian with full width at half maximum $\delta \omega=\Gamma-1 / \tau_{a}$ The coupling matrix $W$ can be eliminated by writıng

$$
\begin{aligned}
& -\pi\left(U^{\dagger} W W^{\dagger} U\right)_{l l}=\operatorname{Im}\left(U^{\dagger} \mathscr{H} U\right)_{l l}=-\frac{\Gamma}{2}\left(U^{\dagger} U\right)_{l l}, \\
& -\pi\left(U^{-1} W W^{\dagger} U^{-1 \dagger}\right)_{l l}=\operatorname{Im}\left(U^{-1} \mathscr{H} U^{-1 \dagger}\right)_{l l}=-\frac{\Gamma}{2}\left(U^{-1} U^{-1 \dagger}\right)_{l l} .
\end{aligned}
$$

The total output current is found by integratıng over frequency,

$$
I=\left(U^{\dagger} U\right)_{l l}\left(U^{-1} U^{-1 \dagger}\right)_{l l} \frac{N_{\text {up }}}{N_{\text {up }}-N_{\text {low }}} \frac{\Gamma^{2}}{\delta \omega} .
$$

Comparison with the Schawlow-Townes value (1 11$)$ shows that

$$
\delta \omega=2 K \frac{N_{\text {up }}}{N_{\text {up }}-N_{\text {low }}} \delta \omega_{\mathrm{ST}},
$$

where the Petermann factor $K$ is identified as

$$
K=\left(U^{\dagger} U\right)_{\| l}\left(U^{-1} U^{-1 \dagger}\right)_{\| l} \geqslant 1
$$

For time-reversal symmetty, we can choose $U^{-1}=U^{\mathrm{T}}$, and find $K=\left[\left(U U^{\dagger}\right)_{l l}\right]^{2}$. The factor of 2 in the relation between $\delta \omega$ and $\delta \omega_{\mathrm{ST}}$ occurs because we have computed the laser line width in the lineal regime just below the threshold, instead of far above 
the threshold The effect of the non-linearities above threshold is to suppress the amplitude fluctuations while leaving the phase fluctuations intact [28], hence the simple factor of two reduction of the line width The factor $N_{\text {up }} /\left(N_{\text {up }}-N_{\text {low }}\right)$ accounts for the extra noise due to an incomplete population inversion The remaining factor $K$ is due to the non-orthogonality of the cavity modes $[3,4]$, since $K=1$ if $U$ is unitary

\section{Single scattering channel}

Relation (2 18) serves as the starting point for a calculation of the statistics of the Petermann factor in an ensemble of chaotic cavities In this section we consider the case $N=1$ of a single scattering channel, for which the coupling matrix $W$ reduces to a vector $\alpha=\left(W_{11}, W_{21}, \quad, W_{M 1}\right)$ The magnitude $|\alpha|^{2}=\left(M \Delta / \pi^{2}\right) w$, where $w \in$ $[0,1]$ is related to the transmission probability $T$ of the single scattering channel by $T=4 w(1+w)^{-2}$ [29] We assume a basis in which $H$ is diagonal (eigenvalues $\omega_{q}$, right eigenvectors $|q\rangle$, left eigenvectors $\langle q|$ ) In this basis the entries $\alpha_{q}$ remain 1eal for $\beta=1$, but become complex numbers for $\beta=2$ Since the eigenvectors $|q\rangle$ point into random directions, and since the fixed length of $\alpha$ becomes an irrelevant constraint in the limit $M \rightarrow \infty$, each real degree of freedom in $\alpha_{q}$ is an independent Gaussian distributed number [14] The squared modulus $\left|\alpha_{q}\right|^{2}$ has probability density

$$
P\left(\left|\alpha_{q}\right|^{2}\right)=\frac{1}{2 \pi\left|\alpha_{q}\right|^{2}}\left(\frac{2 \pi^{3}\left|\alpha_{q}\right|^{2}}{w \Delta}\right)^{\beta / 2} \exp \left[-\frac{\beta \pi^{2}}{2 w \Delta}\left|\alpha_{q}\right|^{2}\right]
$$

Eq (3 1) is a $\chi^{2}$-distribution with $\beta$ degrees of freedom and mean $\Delta w / \pi^{2}$

We first determine the distribution of the decay rate $\Gamma$ of the lasing mode, following Ref [30] Since the lasing mode is the mode closest to the real axis, its decay rate is much smaller than the typical decay rate of a mode, which is $\simeq T \Delta$ Then we calculate the conditional distribution and mean of the Petermann factor for given $\Gamma$ The unconditional distribution of the Petermann factor is found by folding the conditional distribution with the distribution of $\Gamma$, but will not be considered here

\section{Decay rate of the lasing mode}

The amplification with rate $1 / \tau_{a}$ is assumed to be effective over a window $\Omega_{a}=L \Delta$ containing many modes The lasing mode is the mode within this window that has the smallest decay rate $\Gamma$ For such small decay rates we can use first-order perturbation theory to obtain the decay rate of mode $q$,

$$
\Gamma_{q}=2 \pi\left|\alpha_{q}\right|^{2}
$$

The $\chi^{2}$ distribution (3 1) of the squared modulı $\left|\alpha_{q}\right|^{2}$ translates into a $\chi^{2}$ distribution of the decay rates

$$
P(\Gamma) \propto \Gamma^{(2-\beta) / 2} \exp \left(-\frac{\beta \pi \Gamma}{4 w \Delta}\right)
$$


Ignoung correlations, we may obtain the decay rate of the lasing mode by considering the $L$ decay rates as independent random variables diawn from the distribution $P(\Gamma)$ The distribution of the smallest among the $L$ decay rates is then given by

$$
P_{L}(\Gamma)=L P(\Gamma)\left[1-\int_{0}^{\Gamma} \mathrm{d} \Gamma^{\prime} P\left(\Gamma^{\prime}\right)\right]^{L-1}
$$

For small rates $\Gamma$ we can insert distribution (3 3) and obtain

$$
\begin{aligned}
& P_{L}(\Gamma) \approx \frac{1}{\sqrt{\Gamma}} \exp \left(-\frac{L \pi \Gamma}{4 w \Delta}\right)\left[\operatorname{eif}\left(\frac{\pi \Gamma}{4 n \Delta}\right)\right]^{L-1}, \quad \beta=1, \\
& P_{L}(\Gamma) \approx \exp \left(-\frac{L \pi \Gamma}{2 w \Delta}\right), \quad \beta=2
\end{aligned}
$$

Here $\operatorname{erf}(x)=2 \pi^{-1 / 2} \int_{0}^{x} \mathrm{~d} y \exp \left(-y^{2}\right)$ is the error function The decay rate of the lasing mode decreases with increasing width of the amplification window as $\Gamma \sim w \Delta\left(\Omega_{a} / \Delta\right)^{2 / \beta} \ll w \Delta$

\section{Fitst-order perturbation theory}

If the opening is much smaller than a wavelength, then a perturbation theory in $\alpha$ seems a natural starting point We assign the index $l$ to the lasing mode, and write the perturbed right eigenfunction $|l\rangle^{\prime}=\sum_{q} d_{q}|q\rangle$ and the perturbed left eigenfunction $\left\langle\left. l\right|^{\prime}=\sum_{q} e_{q}\langle q|\right.$, in terms of the eigenfunctions of $H$ The coefficients are $d_{q}=U_{q I} / U_{l l}$ and $e_{q}=U_{l q}^{-1} / U_{l l}^{-1}, 1 \mathrm{e}$, we do not normalize the perturbed eigenfunctions but rather choose $d_{l}=e_{l}=1$

To leading order the lasing mode remains at $\Omega=\omega_{I}$ and has width

$$
\Gamma=2 \pi\left|\alpha_{l}\right|^{2}
$$

The coefficients of the wave function are

$$
d_{q}=1 \frac{\pi \alpha_{q} \alpha_{l}^{*}}{\omega_{q}-\omega_{l}}, \quad e_{q}=1 \frac{\pi \alpha_{q}^{*} \alpha_{l}}{\omega_{q}-\omega_{l}}
$$

The Petermann factor of the lasing mode follows from Eq (2 18),

$$
\begin{aligned}
K & =\frac{\left(1+\sum_{q \neq l}\left|d_{q}\right|^{2}\right)\left(1+\sum_{q \neq l}\left|e_{q}\right|^{2}\right)}{\left|1+\sum_{q \neq l} d_{q} e_{q}\right|^{2}} \\
& \approx 1+\sum_{q \neq l}\left|d_{q}-e_{q}^{*}\right|^{2}
\end{aligned}
$$

where we linearized with respect to $\Gamma$ because the lasing mode is close to the real axis From $\mathrm{Eq} \mathrm{(37)} \mathrm{one} \mathrm{finds}$

$$
K=1+\left(2 \pi\left|\alpha_{l}\right|\right)^{2} \sum_{q \neq l} \frac{\left|\alpha_{q}\right|^{2}}{\left(\omega_{l}-\omega_{q}\right)^{2}}
$$

We seek the distibution $P(K)$ and the average $\langle K\rangle_{\Omega \Gamma}$ of $K$ for a given value of $\Omega$ and $\Gamma$ 
For $\beta=1$, the probability to find an eigenvalue at $\omega_{q}$ given that theie is an eigenvalue at $\omega_{l}$ vanishes linearly for small $\left|\omega_{q}-\omega_{l}\right|$, as a consequence of eigenvalue repulsion constramed by time-1eversal symmetry Since expression (39) for $K$ diverges quadratlcally for small $\left|\omega_{q}-\omega_{l}\right|$, we conclude that $\langle K\rangle_{\Omega \Gamma}$ does not exist in perturbation theory ${ }^{1}$ This severely complicates the problem

\section{Summation of the perturbation sertes}

To obtain a finite answer for the average Petermann factor we need to go beyond perturbation theory By a complete summation of the perturbation series we will in this section obtain results that are valid for all values $T \leqslant 1$ of the transmission probability Our starting point aie the exact relations

$$
\begin{aligned}
& d_{q} z_{l}=\omega_{q} d_{q}-1 \pi \alpha_{q} \sum_{p} \alpha_{p}^{*} d_{p}, \\
& e_{q} z_{l}=\omega_{q} e_{q}-1 \pi \alpha_{q}^{*} \sum_{p} \alpha_{p} e_{p},
\end{aligned}
$$

between the complex eigenvalues $z_{q}$ of $\mathscr{H}$ and the real eigenvalues $\omega_{q}$ of $H$ Distinguishing between $q=l$ and $q \neq l$, we obtain thiee recursion telations

$$
\begin{aligned}
& z_{l}=\omega_{l}-1 \pi\left|\alpha_{l}\right|^{2}-1 \pi \alpha_{l} \sum_{q \neq l} \alpha_{q}^{*} d_{q}, \\
& 1 d_{q}=\frac{\pi \sigma_{q}}{z_{l}-\omega_{q}}\left(\alpha_{l}^{*}+\sum_{p \neq l} \alpha_{p}^{*} d_{p}\right), \\
& 1 e_{q}=\frac{\pi \sigma_{q}^{*}}{z_{l}-\omega_{q}}\left(\alpha_{l}+\sum_{p \neq l} \alpha_{p} e_{p}\right)
\end{aligned}
$$

We now use the fact that $z_{l}$ is the eigenvalue closest to the real axis We may therefore assume that $z_{l}$ is close to the unperturbed value $\omega_{l}$ and replace the denominator $z_{l}-\omega_{q}$ in Eq $(311 \mathrm{c})$ by $\omega_{l}-\omega_{q}$ That decouples the recursion ielations, which may then be solved in closed form

$$
\begin{aligned}
& z_{l}=\omega_{l}-1 \pi\left|\alpha_{l}\right|^{2}(1+1 \pi A)^{-1}, \\
& 1 d_{q}=\frac{\pi \alpha_{q} \alpha_{l}^{*}}{\omega_{l}-\omega_{q}}(1+\imath \pi A)^{1}, \\
& 1 e_{q}=\frac{\pi \alpha_{q}^{*} \alpha_{l}}{\omega_{l}-\omega_{q}}(1+1 \pi A)^{-1}
\end{aligned}
$$

\footnotetext{
${ }^{1}$ For bioken time-reversal symmetry thete is no divergence We can use the known two-point cotrelation function $R\left(\omega_{l}, \omega_{q}\right)$ of the Gaussian unitary enscmble to obtain $\langle K\rangle_{\Omega I}=1+\frac{1}{3} \pi T \Gamma / \Delta$ fol $T \ll 1$
} 
We have defined

$$
A=\sum_{q \neq l}\left|\alpha_{q}\right|^{2}\left(\omega_{l}-\omega_{q}\right)^{-1}
$$

The decay rate of the lasing mode is

$$
\Gamma=-2 \operatorname{Im} z_{l}=2 \pi\left|\alpha_{l}\right|^{2}\left(1+\pi^{2} A^{2}\right)^{-1}
$$

From $\mathrm{Eq}(38)$ we find

$$
K=1+\frac{2 \pi \Gamma}{A} \frac{B}{1+\pi^{2} A^{2}},
$$

with

$$
B=\Delta \sum_{q \neq l}\left|\alpha_{q}\right|^{2}\left(\omega_{l}-\omega_{q}\right)^{-2}
$$

The problem is now reduced to a calculation of the jount probability distribution $P(A, B)$ This problem is closely related to the level curvature problem of random-matrix theory [31-33] The calculation is presented in Append1x A The result is

$$
P(A, B)=\frac{\pi}{24}\left(\frac{8}{\pi w}\right)^{\beta / 2} \frac{\left(\pi^{2} A^{2}+w^{2}\right)^{\beta}}{B^{2+3 \beta / 2}} \exp \left[-\frac{\beta w}{2 B}\left(\frac{\pi^{2} A^{2}}{w^{2}}+1\right)\right]
$$

\section{Probability distribution of the Petermann factor}

From Eqs (31), (3 14), (3 15), and (317) we can compute the probability distribution

$$
\begin{aligned}
& P(K)=\langle Z\rangle^{-1}\left\langle\delta\left(K-1-\frac{2 \pi \Gamma}{\Delta} \frac{B}{1+\pi^{2} A^{2}}\right) Z\right\rangle, \\
& Z=\delta\left(\Omega-\omega_{l}\right) \partial\left(\Gamma-\frac{2 \pi\left|\alpha_{l}\right|^{2}}{1+\pi^{2} A^{2}}\right),
\end{aligned}
$$

of $K$ at fixed $\Gamma$ and $\Omega$ by averaging over $\left|\alpha_{I}\right|^{2}, A$, and $B$ In principle one should also requile that the decay rates of modes $q \neq l$ ale bigger than $\Gamma$, but this extra condition becomes irnelevant for $\Gamma \rightarrow 0$ The average of $Z$ over $\left|\alpha_{l}\right|^{2}$ with Eq (3 1) yields a factor $\left(1+\pi^{2} A^{2}\right)^{\beta / 2}$ (Only the behavior of $P\left(\left|\alpha_{l}\right|^{2}\right.$ ) for small $\left|\alpha_{l}\right|^{2}$ matters, because we concentiate on the lasing mode) After integration over $B$ the distribution can be expressed as a ratio of integials ove1 $A$,

$$
\begin{aligned}
P(K)= & \frac{(2 \pi)^{2 \beta}}{3 \beta} \frac{\Delta w}{\Gamma}\left(\frac{(K-1) A}{w \Gamma}\right)^{2-3 \beta / 2} \\
& \times \int_{0}^{\infty} \mathrm{d} A \frac{\left(1+\pi^{2} A^{2} / w^{2}\right)^{\beta}}{\left(1+\pi^{2} A^{2}\right)^{1+\beta}} \exp \left[-\frac{\beta \pi w \Gamma\left(1+\pi^{2} A^{2} / w^{2}\right)}{(K-1) A\left(1+\pi^{2} A^{2}\right)}\right] \\
& \times\left(\int_{0}^{\infty} \mathrm{d} A \frac{\left(1+\pi^{2} A^{2}\right)^{\beta / 2}}{\left(1+\pi^{2} A^{2} / w^{2}\right)^{1 \mid \beta / 2}}\right)^{-1}
\end{aligned}
$$




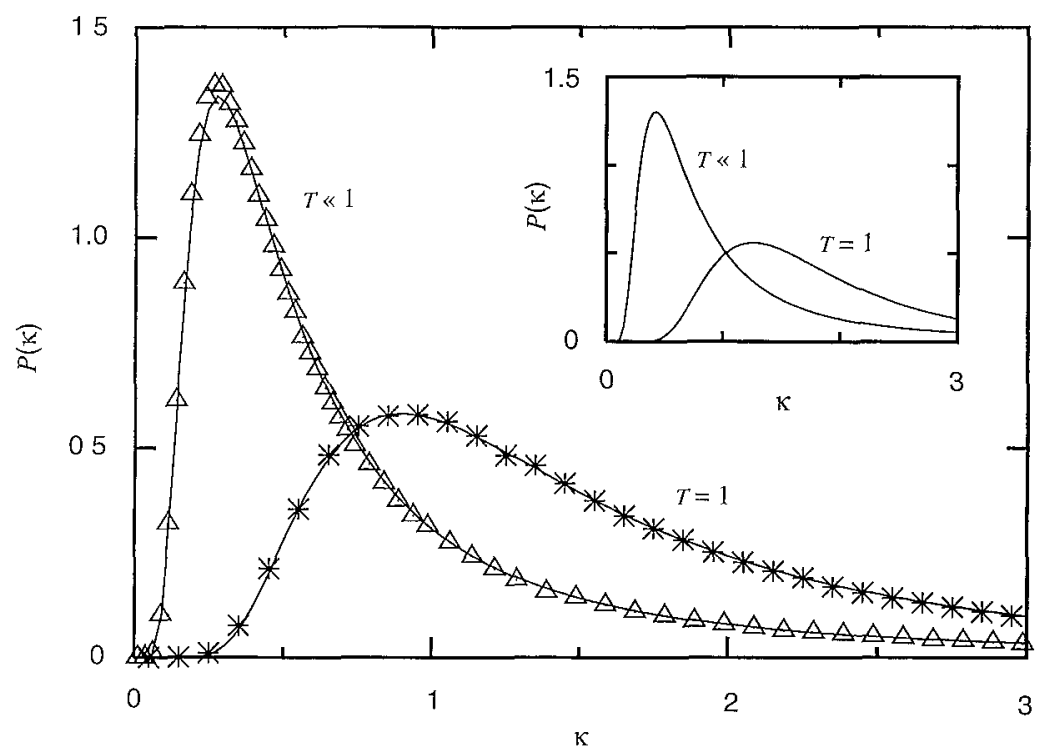

Fig 2 Probability distribution of the rescaled Petermann factor $\kappa=(K-1) \Delta / \Gamma T$ for $T=1$ and $T \ll 1$, in the presence of time-reversal symmetry The solid curves follow fiom Eqs (320) (with $\beta=1$ ) and (3 2la) The data points follow from a numcical simulation of the random-matrix model The inset shows the results (320) (with $\beta=2$ ) and (321b) for broken time-reversal symmetry

We introduce the rescaled Petermann factor $\kappa=(K-1) \Delta / \Gamma T$. A simple result for $P(\kappa)$ follows for $T=1$,

$$
P(\kappa)=\frac{4 \beta \pi^{2 \beta}}{3 \kappa^{2+3 \beta / 2}} \exp \left[-\frac{\beta \pi}{\kappa}\right],
$$

and for $T \ll 1$,

$$
\begin{aligned}
& P(\kappa)=\frac{\pi}{12 \kappa^{2}}\left(1+\frac{\pi}{2 \kappa}\right) \exp \left[-\frac{\pi}{4 \kappa}\right], \quad \beta=1, \\
& P(\kappa)=\frac{\pi}{8 \sqrt{2 \kappa^{5}}}\left(1+\frac{2 \pi}{3 \kappa}+\frac{\pi^{2}}{3 \kappa^{2}}\right) \exp \left[-\frac{\pi}{2 \kappa}\right], \quad \beta=2 .
\end{aligned}
$$

As shown in Fig. 2, the distributions are very broad and asymmetric, with a long tail towards large $\kappa$.

To check our analytical results we have also done a numerical simulation of the random-matrix model, generating a large number of random matrices $H$ and computing $K$ from Eq. (2.18). As one can see from Fig. 2, the agreement with the theoretical predictions is flawless. 


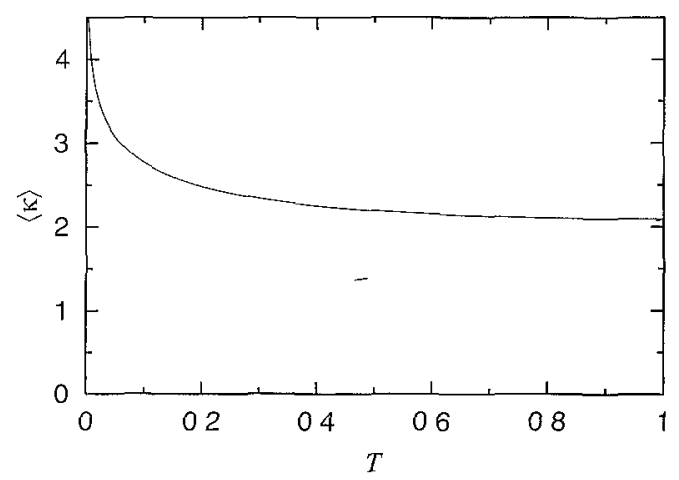

Fig 3 Average of the rescaled Petermann factor $k$ as a function of transmission probability $T$ The solid curve is the result (322) in the presence of timc ieversal symmetry, the dashed curve is the result (324) for broken time reversal symmetry For small $T$ the solid curve diverges $\propto \ln T{ }^{1}$ while the dashed curve has the finte $1 \mathrm{mmit}$ of $\pi / 3$ For $T=1$ both curves 1 cach the valuc $2 \pi / 3$

\section{Mean Petermann factor}

The distıbution (3 19) gives for preserved time-reversal symmetry $(\beta=1)$ the mean Petermann factor

$$
\langle K\rangle_{\Omega \Gamma}=1-\frac{\Gamma}{\Delta} \frac{2 \pi}{3} \frac{G_{22}^{22}\left(\begin{array}{c|cc}
w^{2} & 0 & 0 \\
& -\frac{1}{2} & -\frac{1}{2}
\end{array}\right)}{G_{22}^{22}\left(\begin{array}{l|ll}
w^{2} & -\frac{1}{2} & \frac{1}{2} \\
-1 & 0
\end{array}\right)}
$$

in terms of the 1atio of two Meijer $G$-functions We have plotted the result in Fig 3, as a function of $T=4 w(1+w)^{-2}$

It is remarkable that the average $K$ depends non-analytically on $T$, and hence on the area of the opening (The transmission probability $T$ is related to the area $\mathscr{A}$ of the opening by $T \simeq \mathscr{A}^{3} / \lambda^{6}$ for $T \ll 1[34]$ ) For $T \ll 1$, the average approaches the form

$$
\langle K\rangle_{\Omega \Gamma}=1+\frac{\pi}{6} \frac{T \Gamma}{\Delta} \operatorname{In} \frac{16}{T}
$$

The most probable (or modal) value of $K-1 \simeq T \Gamma / \Delta$ is parametıcally smaller than the mean value $(323)$ for $T \ll 1$ The non-analytıcity results from the relatıvely weak eigenvalue repulsion in the presence of time-reversal symmetry If time-reversal symmetry is broken, then the stronger quadiatic repulsion is sufficient to overcome the $\omega^{-2}$ divergence of perturbation theory (39) and the average $K$ becomes an analytic function of $T$ For this case, we find fiom Eq (3 19) the mean Petermann factor

$$
\langle K\rangle_{\Omega \Gamma}=1+\frac{\Gamma}{\Delta} \frac{4 \pi w}{3\left(1+w^{2}\right)},
$$

shown dashed in Fig 3 


\section{Many scattering channels}

For arbitrary number of scattering channels $N$ the coupling matix $W$ is an $M \times N$ rectangular matix The square matrix $\pi W^{\dagger} W$ has $N$ eigenvalues $(M A / \pi) w_{n}$ The transmission coefficients of the eigenchannels are

$$
T_{n}=\frac{4 w_{n}}{\left(1+w_{n}\right)^{2}}
$$

A single hole of area $\mathscr{A} \gg \lambda^{2}$ (at wavelength $\lambda$ ) corresponds to $N \simeq 2 \pi \mathscr{A} / \lambda^{2}$ fully transmitted scattering channels, with all $T_{n}=w_{n}=1$ the same

As in the single-channel case, we first determine the distribution of the decay rate $\Gamma$ of the lasing mode This decay rate is smaller than the typical decay rate $\Gamma_{0}=T N \Delta / 2 \pi$ of the non-lasing modes Then we calculate the mean Petermann factor $\langle K\rangle$ for given $\Gamma$ and investigate its behavior for the atypically small decay rates of the lasing mode

\section{Decay rate of the lasing mode}

The distribution of decay rates $P(\Gamma)$ has been calculated by Fyodoiov and Sommers For broken tıme-reversal symmetry the result is $[17,18]$

$$
\begin{aligned}
& P(\Gamma)=\frac{\pi}{\Delta} \mathscr{F}_{1}\left(\frac{\pi}{\Delta} \Gamma\right) \mathscr{F}_{2}\left(\frac{\pi}{\Delta} \Gamma\right), \\
& \mathscr{F}_{1}(y)=\frac{1}{2 \pi} \int_{-\infty}^{\infty} \mathrm{d} x \mathrm{e}^{-1 x y} \prod_{n=1}^{N} \frac{1}{g_{n}-1 x}, \\
& \mathscr{F}_{2}(y)=\frac{1}{2} \int_{-1}^{1} \mathrm{~d} x \mathrm{e}^{-x y} \prod_{n=1}^{N}\left(g_{n}+x\right),
\end{aligned}
$$

where $g_{n}=-1+2 / T_{n}$ For identical $g_{n} \equiv g$ the two functions $\mathscr{F}_{1}$ and $\mathscr{F}_{2}$ simplify to

$$
\begin{aligned}
& \mathscr{F}_{1}(y)=\frac{1}{(N-1)^{!}} y^{N-1} \mathrm{e}^{-g y}, \\
& \mathscr{\mathscr { F }}_{2}(y)=\sum_{n=0}^{N}(-1)^{n}\left(\begin{array}{c}
N \\
n
\end{array}\right) g^{N-n} \frac{\mathrm{d}^{n}}{\mathrm{~d} y^{n}}\left(\frac{\sinh y}{y}\right),
\end{aligned}
$$

and a convenient form of the distribution function is

$$
P(\Gamma)=\frac{\Delta}{2 \pi \Gamma^{2}(N-1)^{\dagger}} \int_{N(1-T) \Gamma / \Gamma_{0}}^{N \Gamma / \Gamma_{0}} \mathrm{~d} x x^{N} \mathrm{e}^{-x}
$$

The behavior of $P(\Gamma)$ for various numbers $N$ of fully transmitted $(T=1)$ scattering channels is 1llustrated in Fig 4

The 1esult for preserved time-reversal symmetry is a bit more involved [19] Fortunately, we can draw all important conclusions from the results for broken time-reversal symmetry, on which we will concentrate here 


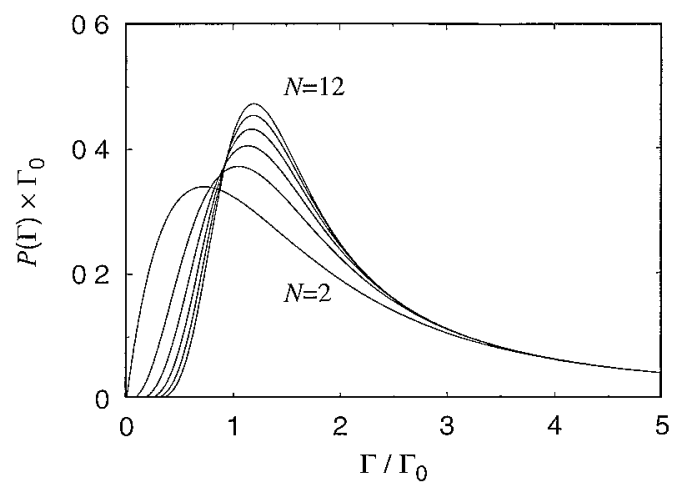

Fig 4 Decay rate distribution $P(\Gamma)$ of a chaotic cavity with an opening that suppotts $N-244681012 \mathrm{ful}$ ly transmitted scattering channels Computed from Eq (42) for the case of bioken time 1cversal symmetry

For large $N$, the distribution $P(\Gamma)$ becomes non-zero only in the interval $\Gamma_{0}<\Gamma<$ $\Gamma_{0} /(1-T)$, where it is equal to $[35,36]$

$$
P(\Gamma)=\frac{\Gamma_{0}}{T \Gamma^{2}}, \quad \Gamma_{0}<\Gamma<\Gamma_{0} /(1-T)
$$

This limit is $\beta$-1ndependent The smallest decay rate $\Gamma_{0}$ conesponds to the inverse mean dwell time in the cavity

We are interested in the "good cavity" regime, where the typical decay rate $\Gamma_{0}$ is small compared to the amplification bandwidth $\Omega_{a}$ From $\Gamma_{0}=T N \Delta / 2 \pi$ it follows that the number $L \simeq \Omega_{a} / \Delta$ of amplified modes is then much larger than $T N$ In this legime the decay 1 ate of the lasing mode (the smallest among the $L$ decay rates in the fiequency window $\Omega_{a}$ ) drops below $\Gamma_{0}$ The asymptotic result (4 5) cannot be used in this case, since it does not describe accurately the tail $\Gamma \lesssim \Gamma_{0}$ Going back to the exact result (42) we find for the tail of the distribution the expression

$$
P(\Gamma)=\frac{\pi}{N T^{2} \Delta}[1+\operatorname{eif}(u)]+\mathcal{O}\left(N^{-3 / 2}\right),
$$

where we have defined $u=\sqrt{N / 2}\left(\Gamma / \Gamma_{0}-1\right)$ The distribution $P_{L}(\Gamma)$ of the lasing mode follows fiom $P(\Gamma)$ by means of $\mathrm{Eq}$ (34) We find that it has a pronounced maximum at a value $u_{\max }$ determined by

$$
\frac{\exp \left(-u_{\max }^{2}\right)}{\left[1+\operatorname{eif}\left(u_{\max }\right)\right]^{2}}=\frac{L-1}{\sqrt{2 N}} \frac{\sqrt{\pi}(g+1)}{4}
$$

For $L \gg \sqrt{N}$ (and hence also in the good cavity regime) we find $u_{\max } \sim-\sqrt{\ln L}<0$, and the deviation of $\Gamma$ from $\Gamma_{0}$ is of ordet $\Delta \sqrt{N} \ll \Gamma_{0}$ (as long as $L \ll \mathrm{e}^{N}$ )

\section{Mean Petermann factor}

E1genfunction correlations of non-Hermitian operators have been studied in Refs [l 20 22] The eigenfunction autoconelator considered in these studies is directly connected to the Petermann factor $K$ Ref [20] provides a convenient expression of 
the mean Petermann factor,

$$
M \pi\langle K\rangle_{\Omega \Gamma} \rho(\omega)=\lim _{i \rightarrow 0^{+}}\left\langle\left(\operatorname{tr} \frac{\varepsilon}{(\omega-\mathscr{H})\left(\omega^{*}-\mathscr{H}^{\dagger}\right)+\varepsilon^{2}}\right)^{2}\right\rangle
$$

In Ref [20] this average has been calculated pertubatively for $N \gg 1$, with the result

$$
\langle K\rangle_{\Omega \Gamma} \approx-N\left(\frac{\Gamma}{\Gamma_{0}}-1\right)\left(\frac{(1-T) \Gamma}{\Gamma_{0}}-1\right)
$$

for $\Gamma_{0}<\Gamma<\Gamma_{0} /(1-T)$ This result is at the same level of appioximation as $\mathrm{Eq}$ (45) for the distribution of the decay rates, $1 \mathrm{e}$, it does not describe the range $\Gamma \lesssim \Gamma_{0}$ of atypically small decay rates Since that is precisely the range that we need for the Petermann factor, we cannot use the existıng perturbatıve results We have calculated the mean Petermann factor non-perturbatively for any $\Gamma$ and $N$, assuming broken time-reversal symmetry The derivation is given in Appendix B The final result for the mean Petermann factor is

$$
\begin{aligned}
& \langle K\rangle_{\Omega \Gamma}=1+\frac{2 S(\pi \Gamma / \Delta)}{\mathscr{\mathscr { F }}_{1}(\pi \Gamma / \Delta) \mathscr{F}_{2}(\pi \Gamma / \Delta)}, \\
& S(y)=-\int_{0}^{y} \mathrm{~d} y^{\prime} \mathscr{F}_{1}\left(y^{\prime}\right) \frac{\partial}{\partial y^{\prime}} \widetilde{\mathscr{F}}_{2}\left(y^{\prime}\right),
\end{aligned}
$$

with $\mathscr{F}_{1}$ and $\mathscr{F}_{2}$ given in Eq (42) For identical $g_{n} \equiv g$ we can use Eq (43) and obtain by successive integrations by parts

$$
S(y)=\sum_{n 0}^{N-1} \frac{(-1)^{n}}{n^{1}} y^{n} \frac{\mathrm{d}^{n}}{\mathrm{~d} y^{n}}\left\{\mathrm{e}^{-q y} \frac{\mathrm{d}}{\mathrm{d} y}\left(\frac{\sinh y}{y}\right)\right\}
$$

For $N=1$ and $\Gamma \ll \Delta$ we recover the single-channel result ( 324 ) of the previous section In what follows we will continue to assume for simplicity that all $q_{n}$ 's are equal to a common value $g$

The large- $N$ behavior can be conveniently studied from the expiession

$$
S(y)=-\frac{1}{4 y^{2}(N-1)^{\prime}} \int_{y(g-1)}^{y(g+1)} \mathrm{d} x x^{N-1} \mathrm{e}^{-\lambda}[x-(g-1) y][x-(g+1) y],
$$

because the integral permits a saddle-point approximation For $\Gamma>\Gamma_{0}$ we recover Eq (49), but now we can also study the piecise behavior of the mean Petermann factor for $\Gamma \lesssim \Gamma_{0}$, hence also for decay rates relevant for the lasing mode The results w1ll again be presented in terms of the rescaled parameter $u=\sqrt{N / 2}\left(\Gamma / \Gamma_{0}-1\right)$ We expand the integrands in Eqs (4 4) and (4 12) around the saddle point at $x=N$ (which coincides with the upper integration limit at $\Gamma=\Gamma_{0}$ ) and keep the first non-Gaussian correction This yields

$$
\begin{aligned}
\langle K\rangle_{\Omega \Gamma}= & T \sqrt{2 N}[F(u)+u]-T(g-1) u^{2} \\
& +T F(u)\left[(3-g) u+\frac{4}{3} u^{3}+\frac{4}{3}\left(1+u^{2}\right) F(u)\right] \\
& +\mathcal{O}\left(N^{-1 / 2}\right),
\end{aligned}
$$




$$
F(u)=\frac{\exp \left(-u^{2}\right)}{\sqrt{\pi}[1+\operatorname{erf}(u)]}
$$

For $\Gamma=\Gamma_{0}(u=0)$ this simplifies to

$$
\langle K\rangle_{\Omega \Gamma \quad \Gamma_{0}}=T\left(\sqrt{\frac{2 N}{\pi}}+\frac{4}{3 \pi}\right)
$$

We see that the mean Petermann factor values on the same scale of $\Gamma$ as the decay-1ate distisbution $P(\Gamma)$, Eq (46) However, while $P(\Gamma)$ decays exponentially for $u \ll-1$, the mean Petermann factor displays an algebraic tail

$$
\langle K\rangle_{\Omega \Gamma}=-\frac{T \sqrt{N}}{u \sqrt{2}}+1-T+\mathscr{O}\left(u^{2}\right)
$$

Fo1 an amplification window $\Omega_{a}=L \Delta$ with $L \gg \sqrt{N}$ we found in Section 41 that the decay rate $\Gamma$ of the lasing mode drops below $\Gamma_{0}$ (the 1escaled parameter $u_{\mathrm{m} \text { ix }} \sim$ $-\sqrt{\ln L})$ Still, the mean Petermann factor

$$
\langle K\rangle_{\Omega \Gamma} \sim \sqrt{\frac{N}{\ln L}}
$$

1emains parametrically laiger than unity (as long as $L \ll \sqrt{N} \mathrm{e}^{N}$ )

We now compare our analytical findings with the iesults of numerical simulations We generated a large number of random matices $\mathscr{H}$ with dimension $M=120(M=200)$ for $N=2,4,6,8(N=10,12)$ fully tiansmitted scatteing channels $(g=T=1)$ Fig 5 shows the mean $K$ at given $\Gamma$ We find excellent agreement with oui analytical result (4 10)

The behavior $\langle K\rangle \sim \sqrt{N}$ at $\Gamma=\Gamma_{0}$ is shown in F1g 6 The inset depicts the disti1bution of $K$ at $\Gamma=\Gamma_{0}$ for $N=10$, which only can be accessed numerically We see that the mean Petermann factor is somewhat latger than the most probable (or modal) value

\section{Preserved tme-reversal symmetry}

In the deivation of the mean Peteimann factor for broken tıme-reveisal symmetry Appendix B) it turned out that the final result is formally connected to the expression for the decay-rate distibution $P(\Gamma)$, in as much as both expressions are built from the factors $\mathscr{T}_{1}$ (involving non-compact bosonic degrees of fieedom of the saddle-point manifold) and $\mathscr{F}_{2}$ (involving compact bosonic degiees of fieedom of that manifold) We tried to translate this description to the case of preseived time-reversal symmetry $(\beta=1)$, by operating in the same way on the compact and non-compact factors of the expression of Ref [19], but could obtain a satisfactory iesult only for $N=2$,

$$
\langle K\rangle=\frac{1}{2 \Gamma_{0}} \frac{\Gamma\left(\Gamma-\Gamma_{0}\right) \exp \left(\Gamma / \Gamma_{0}\right)+\Gamma_{0}^{2} \sinh \left(\Gamma / \Gamma_{0}\right)}{\Gamma \cosh \left(\Gamma / \Gamma_{0}\right)-\Gamma_{0} \sinh \left(\Gamma / \Gamma_{0}\right)}
$$

In Fig 7 this expression is compared to the result of a numerical simulation 


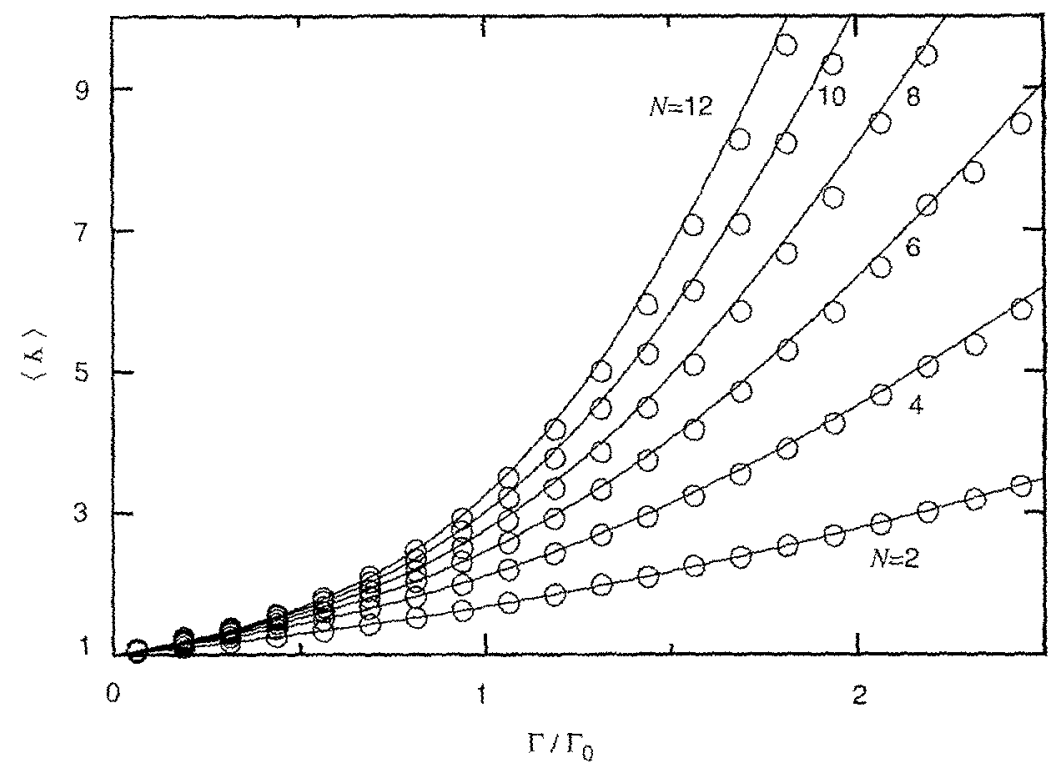

Fig 5 Avalage Petermann factor $\langle K\rangle$ as a function of the decay late $I$ for diflement aalues $\lambda$ of fully transmitcd scatterng channels The soltd curves are the analytical result (4 10) the data ponts ate obtancd by a numencal simulation Imme-levosal symmetry is btoken

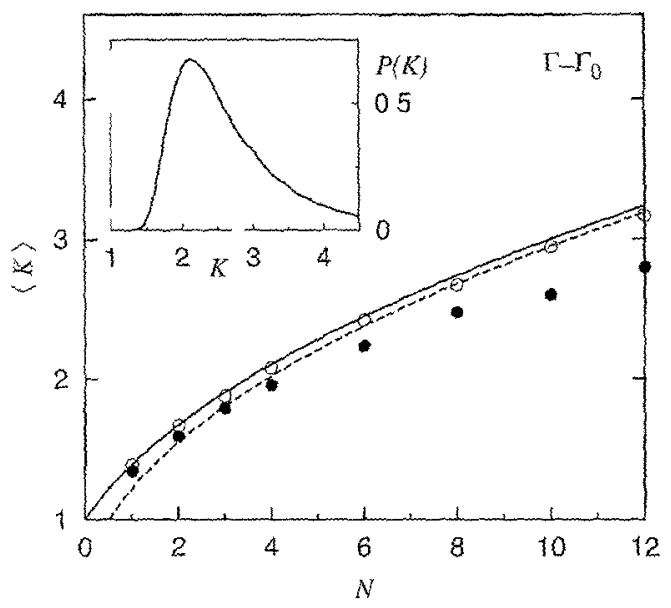

F1g 6 Aretage of the Petermann factor $K$ at $\Gamma-I_{0}$ as function of the number $V$ of fully iransmutted scatrerng chanmels The analytical result $(410)$ to broken tme reversal symmetry (full curre) is compared with the result of a numencal smulation (open circles for broken tme reversal symmety filled cucles for presencd tme reversal symmetry) The dashed hine is the lange $N$ tesult (A 14) The msct shons the distubution of $K$ at $l-\Gamma_{0}$ tor $N=10$ 


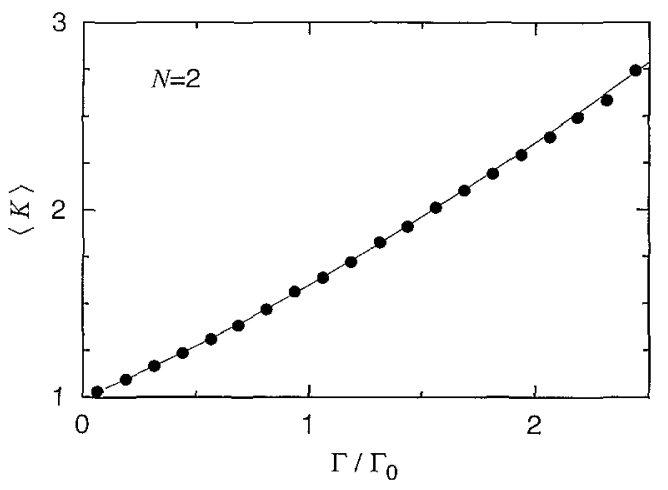

Ftg 7 Theoretical expectation (4 17) (full curve) and the icsult of a numencal simulation (data points) for the average Petermann factor $K$ in the presence of time-reversal symmetry, as a function of the decay rate $\Gamma$ for 2 fully transmitted scattering channels.

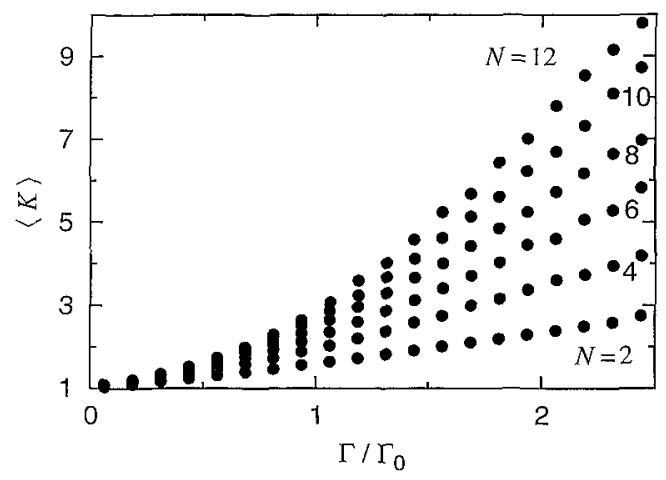

Fig 8 Results of a numerical simulation of the average Peteimam factor $\langle K\rangle$ in the presence of time-1eversal symmetry, as a function of the decay rate $\Gamma$ for $N$ fully tiansmitted scatteing channels

For larger numbers of channels we can draw our conclusions from the numerical results that are presented in Fig. 8. Interestingly enough the data points for $N$ channels are close to the results for broken time-reversal symmetry with $N / 2$ channels, when the decay rate is given in units of $\Gamma_{0}$. This is illustrated for $N=8$ in Fig. 9. Such a rule of thumb (motivated by the number of real degrees of freedom that enter the non-Hermitian part of $\mathscr{H}$ ) was already known for the decay rate distribution (inset in Fig. 9). Hence the Petermann factor for the lasing mode should again display a sublinear growth with increasing channel number $N$. This expectation is indeed confirmed by the numerical simulations, see the filled circles in Fig. 6. 


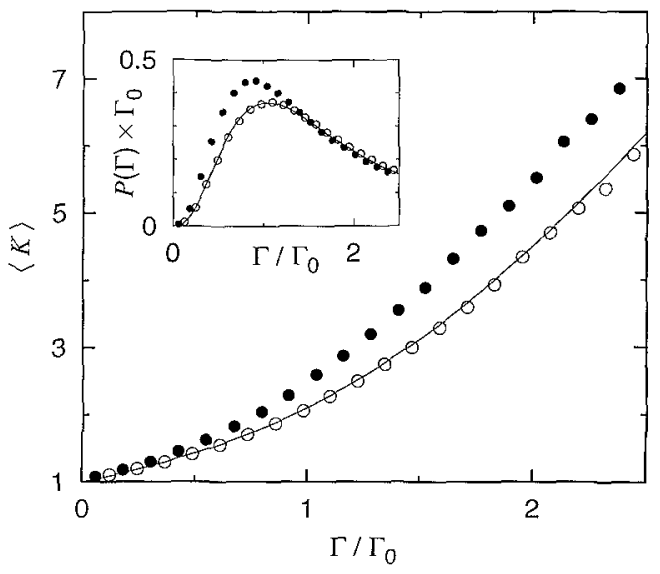

Fig 9 Average Petermann factor $\langle K\rangle$ for $N=4, \beta=2$ [open crrcles result of a numerical simulation, curve Eq (410)] and for $N=8, \beta=1$ (filled circles result of a numencal simulation) The patametcr $\Gamma_{0}$ equals $N \Delta / 2 \pi$ in both cases, so it is twice as laige for $\beta=2$ as for $\beta=1$ The inset depicts the probability distribution of $\Gamma$

\section{Discussion}

The Petermann factor $K$ enters the fundamental lower limit of the laser line width due to vacuum fluctuations and is a measure of the non-orthogonality of cavity modes. We related the Petermann factor to the residue of the scattering-matrix pole that pertains to the lasing mode and computed statistical properties of $K$ in an ensemble of chaotic cavities. The technical complications that had to be overcome arise from the fact that laser action selects a mode which has a small decay rate $\Gamma$, and hence belongs to a pole that lies anomalously close to the real axis. Parametrically large Petermann factors $\propto \sqrt{N}$ arise when the number $N$ of scattering channels is large. For a single scattering channel the mean Petermann factor depends non-analytically on the transmission probability $T$.

The quantity $K$ is also of fundamental significance in the general theory of scattering resonances, where it enters the width-to-height relation of resonance peaks and determines the scattering strength of a quasi-bound state with given decay rate $\Gamma$. If we write the scattering matrix (2.6) in the form

$$
S_{n m}=\delta_{n m}+\sigma_{n} \sigma_{m}^{\prime}(\omega-\Omega+\mathrm{i} \Gamma / 2)^{-1},
$$

then the scattering strengths $\sigma_{n}, \sigma_{m}^{\prime}$ are related to $\Gamma$ by a sum rule. For resonances close to the real axis $(\Gamma \ll \Delta)$ the relation is

$$
\sum_{n, m}\left|\sigma_{n} \sigma_{m}^{\prime}\right|^{2}=\Gamma^{2}
$$

For poles deeper in the complex plane, however, the sum rule has to be replaced by

$$
\sum_{n, m}\left|\sigma_{n} \sigma_{m}^{\prime}\right|^{2}=K \Gamma^{2}, \quad K \geqslant 1
$$


The method of filter diagonalization (or harmonic inveision) that was used in Ref [12] to obtain for the $\mathrm{H}_{3}^{+}$molecular ion the location of poles even deep in the complex plane can also be employed to determine the corresponding residues, and hence $K$

The parameter $K$ defined in Eq (218) appears as a measure of mode nonorthogonality also in problems outside of scattering theory These problems involve non-Hermitian operators that are not of the form (23) [21,22] Many applications share the common feature that they can be addressed statistically by an ensemble description, and that the physically relevant modes lie at the boundary of the complex eigenvalue spectrum The non-perturbative statistical methods reported in this paper should prove useful in the investigation of some of these problems as well

\section{Acknowledgements}

We have benefitted from discussions with P W Brouwer, Y V Fyodorov, and F von Oppen This work was supported by the Nederlandse organisatie voor Wetenschappelık Onderzoek (NWO), the Stıchtıng voor Fundamenteel Onderzoek de1 Mate11e (FOM), and by the European Commission via the Program for the Training and Mobility of Researchers (TMR)

\section{Appendix A. Joint distribution of $A$ and $B$}

We calculate the joint distribution $P(A, B)$ [Eq $(317)]$ of the quantities $A$ [Eq (3 13)] and $B$ [Eq (3 16)] by generalizing the theory of Ref [33] We give the lasing mode $\omega_{l}$ the new index $M$ and assume that 1 lies at the center of the semicucle (22), $\omega_{M}=0$ Other choices just 1enormalize the mean modal spacing $A$, which we can set to $\Delta=1$ The quantities $A$ and $B$ are then of the form

$$
A=\sum_{m-1}^{M-1} \frac{\left|\alpha_{m}\right|^{2}}{\omega_{m}}, \quad B=\sum_{m-1}^{M-1} \frac{\left|\alpha_{m}\right|^{2}}{\omega_{m}^{2}}
$$

The joint piobability distribution of $A$ and $B$,

$$
P(A, B)=\left\langle\delta\left(A-\sum_{m-1}^{M-1} \frac{\left|\alpha_{m}\right|^{2}}{\omega_{m}}\right) \delta\left(B-\sum_{m-1}^{M-1} \frac{\left|\alpha_{m}\right|^{2}}{\omega_{m}^{2}}\right)\right\rangle,
$$

1s obtained by averaging over the variables $\left\{\left|\alpha_{m}\right|^{2}, \omega_{m}\right\}$ The quantities $\left|\alpha_{m}\right|^{2}$ are independent numbers with probability distribution $(31)$ The joint probability distribution of the eigenfrequencies $\left\{\omega_{m}\right\}$ of the closed cavity is the eigenvalue distribution of the Gaussian ensembles (21) of random-matrix theory,

$$
P\left(\left\{\omega_{m}\right\}\right) \propto \prod_{i<j}\left|\omega_{l}-\omega_{j}\right|^{\beta} \exp \left[-\frac{\beta M}{4 l^{2}} \sum_{k} \omega_{k}^{2}\right]
$$

Our choice $\Delta=1$ tianslates into $\mu=M / \pi$ 
The joint probability distribution of the eigenvalues $\left\{\omega_{m}\right\}(m=1, \quad, M-1)$ is found by settıng $\omega_{M}=0 \mathrm{~m} \mathrm{Eq}$ (A 3) It factorizes into the eigenvalue distisbution of $M-1$ dimensional Gaussian matrices $H^{\prime}$ [again distributed according to Eq (21)], and the term $\prod_{j}^{M-1}\left|\omega_{l}\right|^{\beta}=\left|\operatorname{det} H^{\prime}\right|^{\beta}$

In the first step of our calculation, we use the Fourier representation of the $\delta$-functions in $\mathrm{Eq}$ (A 2) and write

$$
\begin{aligned}
P(A, B) \propto & \left\langle\int_{\infty}^{\infty} \mathrm{d} x \int_{-\infty}^{\infty} \mathrm{d} y \mathrm{e}^{1 x A+1 y B} \prod_{m=1}^{M} \int_{0}^{\infty} \mathrm{d}\left|\alpha_{m}\right|^{2} P\left(\left|\alpha_{m}\right|^{2}\right)\right. \\
& \left.\times \exp \left[-1 x \sum_{m 1}^{M} \frac{\left|\alpha_{m}\right|^{2}}{\omega_{m}}-1 y \sum_{m-1}^{M-1} \frac{\left|\alpha_{m}\right|^{2}}{\omega_{m}^{2}}\right]\right\rangle,
\end{aligned}
$$

where the average refers to the variables $\left\{\omega_{m}\right\}$ The integrals ove1 $\left|\alpha_{m}\right|^{2}$ can be performed, resulting in

$$
P(A, B) \propto \int \mathrm{d} x \int \mathrm{d} y \mathrm{e}^{1 x A+1 y B}\left\langle\frac{\operatorname{det} H^{\prime 2 \beta}}{\operatorname{det}\left[H^{\prime 2}+2 \operatorname{tw}\left(x H^{\prime}+y\right) / \pi^{2} \beta\right]^{\beta / 2}}\right\rangle,
$$

where the average is now over the Gaussian ensemble of $H^{\prime}$-matices It is our goal to relate this average to autocorrelators of the secular polynomial of Gaussian distributed random matrices, given in Refs [37,38]

The determinant in the denominator can be expressed as a Gaussian integral,

$$
\begin{aligned}
P(A, B) \propto & \int \mathrm{d} x \int \mathrm{d} y \mathrm{e}^{1 \lambda A+1 y B} \int \mathrm{d} z \int \mathrm{d} H^{\prime} \operatorname{det} H^{\prime 2 \beta} \\
& \times \exp \left[-\frac{\beta \pi^{2}}{4 M} \operatorname{tr} H^{\prime 2}-\mathbf{z}^{\dagger}\left(H^{\prime 2}+\frac{21 w}{\beta \pi^{2}}\left(x H^{\prime}+y\right)\right) \mathbf{z}\right],
\end{aligned}
$$

where the $M-1$ dimensional vector $\mathbf{z}$ is real (complex) for $\beta=1$ (2) Since our original expression did only depend on the eigenvalues of $H^{\prime}$, the formulation above is invariant under orthogonal (unitary) transformations of $H^{\prime}$, and we can choose a basis in which $\mathbf{z}$ points into the direction of the last basis vector (1ndex $M-1$ ) Let us denote the Hamiltonian in the block form

$$
H^{\prime}=\left(\begin{array}{cc}
V & \mathbf{h} \\
\mathbf{h}^{\dagger} & q
\end{array}\right)
$$

Here $V$ is a $(M-2) \times(M-2)$ matrix, $g$ a number, and $\mathbf{h}$ a $(M-2)$ dimensional vector In this notation,

$$
\begin{aligned}
P(A, B) \circ & \int \mathrm{d} x \int \mathrm{d} y \mathrm{e}^{1 x A+1 y B} \int \mathrm{d} \mathbf{z} \int \mathrm{d} g \int \mathrm{d} V \int \mathrm{d} \mathbf{h} \\
& \times \operatorname{det}\left[V^{2 \beta}\left(g-\mathbf{h}^{\dagger} V{ }^{1} \mathbf{h}\right)^{2 \beta}\right] \\
& \times \exp \left[-\frac{\beta \pi^{2}}{4 M}\left(g^{2}+2|\mathbf{h}|^{2}+\operatorname{tr} V^{2}\right)\right] \\
& \times \exp \left[-|\mathbf{z}|^{2}\left(g^{2}+|\mathbf{h}|^{2}+\frac{21 w}{\beta \pi^{2}}(x g+y)\right)\right]
\end{aligned}
$$


The integrals over $x$ and $y$ give $\delta$-functions,

$$
\begin{aligned}
P(A, B) \propto & \int \mathrm{d} \mathbf{z} \int \mathrm{d} g \int \mathrm{d} V \int \mathrm{d} \mathbf{h} \operatorname{det}\left[V^{2 \beta}\left(g-\mathbf{h}^{\dagger} V^{-1} \mathbf{h}\right)^{2 \beta}\right] \\
& \times \exp \left[-\frac{\beta \pi^{2}}{4 M}\left(g^{2}+2|\mathbf{h}|^{2}+\operatorname{tr} V^{2}\right)-|\mathbf{z}|^{2}\left(g^{2}+|\mathbf{h}|^{2}\right)\right] \\
& \times \delta(A-g B) \delta\left(B-2 w|\mathbf{z}|^{2} / \beta \pi^{2}\right)
\end{aligned}
$$

We then integrate over $g$ and $\mathbf{z}$,

$$
\begin{aligned}
P(A, B) \propto & \int \mathrm{d} V \mathrm{~d} \boldsymbol{h} \operatorname{det}\left[V^{2 \beta}\left(\frac{A}{B}-\boldsymbol{h}^{\dagger} V^{-1} \boldsymbol{h}\right)^{2 \beta}\right] B^{(\beta / 2)(M-1)-2} \\
& \times \exp \left[-\frac{\beta \pi^{2}}{4 M}\left(2|\boldsymbol{h}|^{2}+\operatorname{tr} V^{2}\right)-\frac{\beta \pi^{2} B}{2 w}\left(\frac{A^{2}}{B^{2}}+|\boldsymbol{h}|^{2}\right)\right] .
\end{aligned}
$$

We already anticipated $B \gg 1 / M$ and omitted in the exponent a term $-\beta \pi^{2} A^{2} / 4 M B^{2}$

The integial over $\boldsymbol{h}$ can be interpreted as an average over Gaussian random variables with variance

$$
h^{2} \equiv\left\langle\left|h_{l}\right|^{2}\right\rangle=\frac{1}{\pi^{2}} \frac{1}{B / w+1 / M} \approx \frac{w}{\pi^{2} B}\left(1-\frac{w}{M B}\right) .
$$

For the stochastic interpretation one also has to supply the normalization constants proportional to

$$
h^{\beta(M-2)}=\left(\frac{w}{\pi^{2} B}\right)^{\beta(M-2) / 2} \exp \left[-\frac{\beta w}{2 B}\right] .
$$

The integral over $V$ is another Gaussian average, and thus

$$
\begin{aligned}
& P(A, B) \propto Q_{\beta} B^{(\beta / 2)-2} \exp \left[-\frac{\beta w}{2 B}\left(1+\frac{\pi^{2} A^{2}}{w^{2}}\right)\right], \\
& Q_{\beta}=\left\langle\operatorname{det}\left[V^{2 \beta}\left(\frac{A}{B}-\boldsymbol{h}^{\dagger} V^{-1} \boldsymbol{h}\right)^{2 \beta}\right]\right\rangle .
\end{aligned}
$$

After averaging over $\boldsymbol{h}$, one has now to consider for $\beta=1$

$$
Q_{1}=\left\langle\operatorname{det}\left[V^{2} \frac{A^{2}}{B^{2}}+h^{4} V^{2}\left[\left(\operatorname{tr} V^{-1}\right)^{2}+2 \operatorname{tr} V^{-2}\right]\right]\right\rangle,
$$

where only the even terms in $V$ have been kept. The ratio of coefficients in this polynomial in $A / B$ can be calculated from the autocorrelator [38]

$$
\begin{aligned}
G_{1}\left(\omega, \omega^{\prime}\right) & =\frac{\left\langle\operatorname{det}(V+\omega)\left(V+\omega^{\prime}\right)\right\rangle}{\left\langle\operatorname{det} V^{2}\right\rangle} \\
& =-\left.\frac{3}{\pi^{2} x} \frac{\mathrm{d}}{\mathrm{d} x} \frac{\sin \pi x}{\pi x}\right|_{r=\omega-\omega^{\prime}}
\end{aligned}
$$


of the secular polynomial of Gaussian distributed real matrices $V$. This is achieved by expressing the products of traces and determinants through secular coefficients, and these then as derivatives of the secular determinant,

$$
\begin{aligned}
\frac{\left\langle\operatorname{det} V^{2}\left(\operatorname{tr} V^{-1}\right)^{2}\right\rangle}{\left\langle\operatorname{det} V^{2}\right\rangle} & =\left.\frac{\partial^{2}}{\partial \omega \partial \omega^{\prime}} G_{1}\left(\omega, \omega^{\prime}\right)\right|_{\omega=\omega^{\prime}=0} \\
& =-\left.\frac{\partial^{2}}{\partial \omega^{2}} G_{1}(\omega, 0)\right|_{\omega=0}=\frac{\pi^{2}}{5},
\end{aligned}
$$

$$
\frac{2\left\langle\operatorname{det} V^{2}\left(\operatorname{tr} V^{-2}\right)\right\rangle}{\left\langle\operatorname{det} V^{2}\right\rangle}=-\left.4 \frac{\partial^{2}}{\partial \omega^{2}} G_{1}(\omega, 0)\right|_{\omega=0} .
$$

[We used the translational invariance of $G\left(\omega, \omega^{\prime}\right)$.] Eqs. (A.11) and (A.15) yield

$$
Q_{1} \propto \frac{A^{2}}{B^{2}}+\frac{w^{2}}{\pi^{2} B^{2}} .
$$

For $\beta=2$, the average over $\mathbf{h}$ yields the expression

$$
\begin{aligned}
& Q_{2} \propto \frac{A^{4}}{B^{4}}+q_{1} h^{4} \frac{A^{2}}{B^{2}}+q_{2} h^{8}, \\
& q_{1}=6\left\langle\operatorname{det} V^{4}\left[\left(\operatorname{tr} V^{-1}\right)^{2}+\operatorname{tr} V^{-2}\right]\right\rangle, \\
& q_{2}=\left\langle\operatorname { d e t } V ^ { 4 } \left[(\operatorname{tr} V)^{-4}+6 \operatorname{tr} V^{-2}\left(\operatorname{tr} V^{-1}\right)^{2}\right.\right. \\
&\left.\left.+8 \operatorname{tr} V^{-1} \operatorname{tr} V^{-3}+6 \operatorname{tr} V^{-4}+3\left(\operatorname{tr} V^{-2}\right)^{2}\right]\right\rangle .
\end{aligned}
$$

The coefficients can now be computed from the four-point correlator of the Gaussian unitary ensemble [37]:

$$
\begin{aligned}
& G_{2}\left(\omega_{1}, \omega_{2}, \omega_{3}, \omega_{4}\right) \\
&= \frac{\left\langle\operatorname{det}\left(V+\omega_{1}\right)\left(V+\omega_{2}\right)\left(V+\omega_{3}\right)\left(V+\omega_{4}\right)\right\rangle}{\left\langle\operatorname{det} V^{4}\right\rangle} \\
&= \frac{3}{2 \pi^{4}}\left[\frac{\cos \pi\left(\omega_{1}+\omega_{2}-\omega_{3}-\omega_{4}\right)}{\left(\omega_{1}-\omega_{3}\right)\left(\omega_{1}-\omega_{4}\right)\left(\omega_{2}-\omega_{3}\right)\left(\omega_{2}-\omega_{4}\right)}\right. \\
&+\frac{\cos \pi\left(\omega_{1}+\omega_{3}-\omega_{2}-\omega_{4}\right)}{\left(\omega_{1}-\omega_{2}\right)\left(\omega_{1}-\omega_{4}\right)\left(\omega_{3}-\omega_{2}\right)\left(\omega_{3}-\omega_{4}\right)} \\
&\left.+\frac{\cos \pi\left(\omega_{1}+\omega_{4}-\omega_{3}-\omega_{2}\right)}{\left(\omega_{1}-\omega_{3}\right)\left(\omega_{1}-\omega_{2}\right)\left(\omega_{4}-\omega_{3}\right)\left(\omega_{4}-\omega_{2}\right)}\right], \\
& G_{2}(\omega, 0,0,0)=\frac{3}{\pi^{3} \omega^{3}}\left(\sin \pi \omega^{2}-\pi \omega \cos \pi \omega^{2}\right), \\
& G_{2}(\omega, \omega, 0,0)=\frac{3}{2 \pi^{4} \omega^{4}}\left(\cos 2 \pi \omega-1+2 \pi^{2} \omega^{2}\right) .
\end{aligned}
$$


In this case

$$
\begin{aligned}
q_{1} & =\left.\frac{\partial^{2}}{\partial \omega^{2}}\left[6 G_{2}(\omega, \omega, 0,0)-18 G_{2}(\omega, 0,0,0)\right]\right|_{\omega=0} \\
& =2 \pi^{2}, \\
q_{2} & =\left.\frac{\partial^{4}}{\partial \omega^{4}}\left[10 G_{2}(\omega, \omega, 0,0)-15 G_{2}(\omega, 0,0,0)\right]\right|_{\omega=0} \\
& =\pi^{2},
\end{aligned}
$$

which gives

$$
Q_{2} \propto Q_{1}^{2}
$$

Collecting results we obtain Eq. (3.17), where we also included the normalization constant.

\section{Appendix B. Derivation of Eq. (4.10) for the mean Petermann factor}

The computation of the mean Petermann factor from expression (4.8) is facilitated by the fact that it can be obtained from the same generating function $[18,39]$,

$$
\Psi\left(\omega_{1}, \omega_{2}, u_{1}, u_{2}, \varepsilon\right)=\left\langle\frac{\operatorname{det}\left[(\omega-\mathscr{H})\left(\omega^{*}-\mathscr{H}^{\dagger}\right)-\left(u_{1}-\mathrm{i} \varepsilon\right)\left(u_{2}-\mathrm{i} \varepsilon\right)\right]}{\operatorname{det}\left[(\omega-\mathscr{H})\left(\omega^{*}-\mathscr{H}^{\dagger}\right)-\left(u_{1}+\mathrm{i} \varepsilon\right)\left(u_{2}+\mathrm{i} \varepsilon\right)\right]}\right\rangle,
$$

as the distribution function

$$
\rho(\omega)=\lim _{\varepsilon \rightarrow 0^{+}}\left\langle\operatorname{tr} \frac{\varepsilon}{\left(\omega^{*}-\mathscr{H}^{\dagger}\right)(\omega-\mathscr{H})+\varepsilon^{2}} \frac{\varepsilon}{(\omega-\mathscr{H})\left(\omega^{*}-\mathscr{H}^{\dagger}\right)+\varepsilon^{2}}\right\rangle
$$

of poles in the complex plane. (The distribution of poles is related to the distribution of decay rates by $P(\Gamma)=\left.\frac{1}{2} \Delta \rho(\omega)\right|_{\omega=\Omega-\mathrm{i} \Gamma / 2}$.) The relations are

$$
\begin{aligned}
& \pi \rho(\omega)=\lim _{\varepsilon \rightarrow 0^{+}}\left(\frac{\partial^{2}}{\partial \omega_{2} \partial \omega_{2}^{*}}+\frac{1}{2} \frac{\partial^{2}}{\partial \omega_{2} \partial \omega_{1}^{*}}+\frac{1}{2} \frac{\partial^{2}}{\partial \omega_{1} \partial \omega_{2}^{*}}\right) \\
& \quad \times\left.\Psi\left(\omega_{1}, \omega_{2}, 0,0, \varepsilon\right)\right|_{\omega_{1}=\omega_{2}=\omega} \\
& M \pi\langle K\rangle_{\Omega, \Gamma} \rho(\omega)=-\left.\lim _{\varepsilon \rightarrow 0^{+}} \frac{1}{4} \frac{\partial}{\partial u_{1}} \frac{\partial}{\partial u_{2}} \Psi\left(\omega, \omega, u_{1}, u_{2}, \varepsilon\right)\right|_{u_{1}=u_{2}=0}
\end{aligned}
$$

Most of the analysis runs therefore in parallel with the calculation of $\rho(\omega)$ in Ref. [18]. We restrict ourselves to the case of broken time-reversal symmetry, where the algebra is less involved. 
The ratio of determinants in Eq (B 1) can be written as a superdeterminant, which in tuin can be expiessed as a Gaussian integral over bosonic and fermionic valiables

$$
\Psi\left(\omega, \omega, \varepsilon, u_{1}, u_{2}\right)=(-1)^{M}\left\langle\operatorname{Sdet}^{-1}(A)\right\rangle=(-1)^{M}\left\langle\int \mathrm{d} \boldsymbol{\Psi}+\int \mathrm{d} \boldsymbol{\Psi} \mathrm{e}^{\mathbf{i}^{\dagger} A \boldsymbol{\Psi}}\right\rangle
$$

The matrix $A$ is

$$
\begin{aligned}
A= & \left(\begin{array}{cccc}
\omega-\mathscr{H} & 0 & 1 \varepsilon+u_{1} & 0 \\
0 & \omega-\mathscr{H} & 0 & -1 \varepsilon+u_{1} \\
-1 \varepsilon-u_{2} & 0 & -\omega^{*}+\mathscr{H} & 0 \\
0 & -1 \varepsilon+u_{2} & 0 & \omega^{*}-\mathscr{H}^{\dagger}
\end{array}\right) \\
= & (\Omega-H) \otimes \hat{L}+1\left(\pi W^{\dagger} W-\frac{\Gamma}{2}\right) \otimes \hat{\sigma}_{z} \hat{L} \\
& -1 \varepsilon \otimes \hat{\sigma}_{x} \hat{L}+\hat{u} \hat{\sigma}_{x} \hat{L}
\end{aligned}
$$

The vector $\boldsymbol{\Psi}=\boldsymbol{\Psi}_{1} \oplus \boldsymbol{\Psi}_{2} \oplus \boldsymbol{\Psi}_{3} \oplus \boldsymbol{\Psi}_{4}$ is a $4 M$-dimensional supervector consisting of two $M$-dimensional bosonic entries $\Psi_{\alpha}$ with $\alpha=1$ and 3 , supplemented by two $M$-dimensional fermionic entries with $\alpha=2$ and 4 We encounter the four-dimensional supermatrices $\hat{L}=\operatorname{diag}(1,1,-1,1), \hat{u}=\operatorname{diag}\left(-u_{1}, u_{1},-u_{2}, u_{2}\right)$, and $\hat{\sigma}_{l}=\sigma_{1} \otimes \mathbb{1}_{2}$, where $\sigma_{l}$ ate the usual Pauli matrices [e g $\hat{\sigma}_{z}=\operatorname{diag}(1,1,-1,-1)$ ]

The linear appearance of $H$ in the exponent of $\mathrm{Eq}$ (B 5) facilitates the ensemble average with the distribution function (21), since the integial over the independent components of $H$ factorizes, and each single integral is Gaussian The result is

$$
\begin{aligned}
& \left\langle\exp \left[-{ }_{1} \boldsymbol{\Psi}^{\dagger} H \otimes \hat{L} \boldsymbol{\Psi}\right]\right\rangle=\exp \left[-\frac{\mu^{2} M}{2} \operatorname{Str}(\hat{L} \hat{R})^{2}\right], \\
& \hat{R}_{\alpha \beta}=\frac{1}{M} \boldsymbol{\Psi}_{\alpha} \boldsymbol{\Psi}_{\beta}^{\dagger}
\end{aligned}
$$

The order of $\hat{R}$ in the exponent is ieduced from quadratic to lineal by a HubbaidStratonovich transformation, based on the identity

$$
\exp \left[-\frac{\mu^{2} M}{2} \operatorname{Str}(\hat{L} \hat{R})^{2}\right]=\int \mathrm{d} \hat{S} \exp \left[-M \operatorname{Str}\left(\frac{\hat{S}^{2}}{2}-1 \mu \hat{S} \hat{L} \hat{R}\right)\right]
$$

The integral over $\boldsymbol{\Psi}$ and $\boldsymbol{\Psi}^{\dagger}$ is again Gaussian and results in

$$
\begin{aligned}
& \Psi=\int \mathrm{d} \hat{S} \exp \left[-M \operatorname{Str}\left(\frac{\hat{S}^{2}}{2}+\ln \hat{S}\right)\right] \operatorname{Sdet}^{-1}(1+C), \\
& C=\left(\Omega+1\left(\pi W^{\dagger} W-\frac{\Gamma}{2}\right) \otimes \hat{\sigma}_{z}-1 \varepsilon \hat{\sigma}_{x}+\hat{u} \hat{\sigma}_{x}\right) \frac{1}{\mu \hat{S}}
\end{aligned}
$$

One now can write $\operatorname{Sdet}^{-1}(1+C)=\exp [-\operatorname{Str} \ln (1+C)]$ and expand the logarithm to first order in $\Gamma, \varepsilon$, and the source teim $J$, in addition we set $\Omega=0$ and pass from the 
genelating function to the mean Petermann factor according to Eq (B 4) This gives

$$
\begin{aligned}
M \pi\langle K\rangle_{\Omega \Gamma} \rho(\omega)= & -\frac{1}{4} \frac{\pi^{2}}{\Delta^{2}} \int \mathrm{d} \hat{S} \exp \left[-M \operatorname{Str}\left(\frac{\hat{S}^{2}}{2}+\ln \hat{S}\right)\right. \\
& \left.+1 \frac{y}{2} \operatorname{Str} \hat{\sigma}_{L} \hat{S}^{-1}+1 \frac{\varepsilon^{\prime}}{2} \operatorname{Str} \hat{\sigma}_{\lambda} \hat{S}^{-1}\right] \\
& \times \mathrm{t1}_{12} \hat{\sigma}_{x} \hat{S}^{1} \operatorname{tin}_{34} \hat{\sigma}_{\lambda} \hat{S}{ }^{1} \prod_{n-1}^{N} \operatorname{Sdet}^{-1}\left(\mathbb{1}_{4}+1 w_{n} \hat{\sigma}_{z} \hat{S}^{-1}\right)
\end{aligned}
$$

The traces $\mathrm{ti}_{l l} A=A_{l l}+A_{J j}$ operate only on the indicated subspaces We introduced the 1escaled valiables $y=-2 \pi \operatorname{Im} \omega / \Delta=\pi \Gamma / \Delta$ and $\varepsilon^{\prime}=2 \pi \varepsilon / \Delta$ In what follows we will Write $\varepsilon$ instead of $\varepsilon^{\prime}$

The condition $M \gg 1$ justifies a saddle-point appioximation The main contibution to the preceding integral comes from points for which the first part of the exponent is minimal, that is from the solutions of

$$
\frac{1}{\hat{S}}+\hat{S}=0 \Leftrightarrow \hat{S}^{2}=-1
$$

With $\hat{S}=1 \hat{Q}$, the solutions fulfill $\hat{Q}^{2}=1$ As inherited fiom the definition of $\hat{R}$ in Eq (B 7b), $\hat{Q} \hat{L}$ is a Hermitian matrix and $\hat{Q}=\hat{T}^{-1} \hat{Q}_{\mathrm{d} \text { ag }} \hat{T}$ can be diagonalized by a pseudounitary supermatix $\hat{T} \in \mathrm{U}(1,1 / 2)$ (these matrices fulfill $\hat{T}^{\dagger} \hat{L} \hat{T}=\hat{L}$ ) The la1gest manifold which respects the definiteness requirements on $\hat{Q}$ is obtained by the chorce $\hat{Q}_{\mathrm{d} 1 \mathrm{~g}}=\hat{\sigma}_{z}$ Howeve1, rotations in the block $\alpha=1,3$ and in the block $\alpha=2,4$ leave $\hat{Q}$ invaliant, the saddle-point manifold is hence covered exactly once if we take the $\hat{T}$ mat1ices from the coset space $U(1,1 / 2) / U(1 / 1) \times U(1 / 1)$

A convenient parameterization of the coset space has been given by Efetov [40],

$$
\begin{aligned}
& \hat{T}=\left(\begin{array}{cc}
U^{-1} & 0 \\
0 & V^{-1}
\end{array}\right) \exp \left(\begin{array}{cc}
0 & \frac{1}{2} \operatorname{diag}\left(\theta_{1}, 1 \theta_{2}\right) \\
{ }_{2} \operatorname{diag}\left(0_{1}, 10_{2}\right) & 0
\end{array}\right)\left(\begin{array}{cc}
U & 0 \\
0 & V
\end{array}\right), \\
& U=\left(\begin{array}{cc}
\mathrm{e}^{\mathrm{l} \phi} & 0 \\
0 & \mathrm{e}^{1 \phi_{2}}
\end{array}\right)\left(\begin{array}{cc}
1+\rho \rho^{*} / 2 & \rho \\
\rho^{*} & 1+\rho^{*} \rho / 2
\end{array}\right), \\
& V=\left(\begin{array}{cc}
1-\sigma \sigma^{*} / 2 & 1 \sigma \\
1 \sigma^{*} & 1-\sigma^{*} \sigma / 2
\end{array}\right),
\end{aligned}
$$

with bosonic variables $\theta_{1}, 0_{2}, \phi_{1}$, and $\phi_{2}$, and fermionic variables $\rho, \rho^{*}, \sigma$, and $\sigma^{*}$ We introduce $\lambda_{1}=\cosh \theta_{1}$ and $\lambda_{2}=\cos \theta_{2}$ In this parameterization

$$
\begin{aligned}
\text { Str } \hat{\sigma}_{z} \hat{Q}= & 2\left(\lambda_{1}-\lambda_{2}\right), \\
\text { Stt } \hat{\sigma}_{1} \hat{Q}= & -\sinh 0_{1} \mathrm{e}^{1 \phi_{1}}\left[\left(1+\rho \rho^{*} / 2\right)\left(1-\sigma \sigma^{*} / 2\right)-1 \rho \sigma^{*}\right] \\
& +\sinh 0_{1} \mathrm{e}^{-1 \phi_{1}}\left[\left(1+\rho \rho^{*} / 2\right)\left(1-\sigma \sigma^{*} / 2\right)-1 \sigma \rho^{*}\right] \\
& +1 \sin 0_{2} \mathrm{e}^{1 \phi_{2}}\left[\left(1+\rho^{*} \rho / 2\right)\left(1-\sigma^{*} \sigma / 2\right)-1 \rho^{*} \sigma\right] \\
& -1 \sin \theta_{2} \mathrm{e}^{-1 \phi_{2}}\left[\left(1+\rho^{*} \rho / 2\right)\left(1-\sigma^{*} \sigma / 2\right)-1 \sigma^{*} \rho\right],
\end{aligned}
$$




$$
\begin{aligned}
& \operatorname{tr}_{12} \sigma_{x} \hat{Q}=-\sinh \theta_{1} \mathrm{e}^{1 \phi_{1}}\left[\left(1+\rho \rho^{*} / 2\right)\left(1-\sigma \sigma^{*} / 2\right)-\mathrm{i} \sigma^{*} \rho\right] \\
&-\mathrm{i} \sin \theta_{2} \mathrm{e}^{1 \phi_{2}}\left[\left(1+\rho^{*} \rho / 2\right)\left(1-\sigma^{*} \sigma / 2\right)-\mathrm{i} \sigma \rho^{*}\right], \\
& \operatorname{tr}_{34} \sigma_{x} \hat{Q}= \sinh \theta_{1} \mathrm{e}^{-1 \phi_{1}}\left[\left(1+\rho \rho^{*} / 2\right)\left(1-\sigma \sigma^{*} / 2\right)-\mathrm{i} \rho^{*} \sigma\right] \\
&+\mathrm{i} \sin \theta_{2} \mathrm{e}^{-1 \phi_{2}}\left[\left(1+\rho^{*} \rho / 2\right)\left(1-\sigma^{*} \sigma / 2\right)-\mathrm{i} \rho \sigma^{*}\right], \\
& \operatorname{Sdet}^{-1}\left[\mathbb{1}_{4}+w_{n} \hat{\sigma}_{z} \hat{Q}\right]=\frac{g_{n}+\lambda_{2}}{g_{n}+\lambda_{1}} .
\end{aligned}
$$

The integration measure is

$$
\mathrm{d} \hat{Q}=\frac{\mathrm{d} \lambda_{1} \mathrm{~d} \lambda_{2} \mathrm{~d} \phi_{1} \mathrm{~d} \phi_{2} \mathrm{~d} \rho^{*} \mathrm{~d} \rho \mathrm{d} i \sigma^{*} \mathrm{~d} i \sigma}{(2 \pi)^{2}\left(\lambda_{1}-\lambda_{2}\right)^{2}}
$$

In order to integrate over the fermionic variables we have to expand in these quantities and only keep the term in which all four variables appear linearly. The angle $\phi_{2}$ appears in the pre-exponential factor as well as in the exponential term $\exp \left(-\varepsilon \sin \theta_{2} \sin \phi_{2}\right)$. We expand the exponential and integrate over $\phi_{2}$. Only terms of order $\varepsilon^{n} \sinh ^{m} \theta_{1}$ with $n \leqslant m$ survive the limit $\varepsilon \rightarrow 0$. We discard all other terms and obtain

$$
\begin{aligned}
-4 & \frac{\Delta^{2}}{\pi^{2}}\langle K\rangle_{\Omega, \Gamma} \rho(\omega) \\
= & \lim _{\varepsilon \rightarrow 0} \int_{1}^{\infty} \mathrm{d} \lambda_{1} \int_{-1}^{1} \mathrm{~d} \lambda_{2} \frac{1}{\left(\lambda_{1}-\lambda_{2}\right)^{2}} \int_{0}^{2 \pi} \frac{\mathrm{d} \phi_{1}}{2 \pi} D \\
& \times \exp \left[-\mathrm{i} \varepsilon \sqrt{\lambda_{1}^{2}-1} \sin \phi_{1}+y\left(\lambda_{1}-\lambda_{2}\right)\right] \prod_{n=1}^{N} \frac{g_{n}+\lambda_{2}}{g_{n}+\lambda_{1}}, \\
D= & -\mathrm{i} \varepsilon \sinh 0_{1} \sin \phi_{1}\left(2 \sin ^{2} \theta_{2}+\frac{9}{4} \sinh ^{2} \theta_{1}\right) \\
& +\frac{\varepsilon^{2}}{4} \sinh 0_{1}\left[\sinh 0_{1} \cos ^{2} \phi_{1}-\left(3 \cos ^{2} \phi_{1}+5 \sin ^{2} \phi_{1}\right) \sin ^{2} \theta_{2}\right] \\
& +\mathrm{i} \varepsilon^{3} \sinh ^{3} 0_{1} \sin _{1} \sin ^{2} \theta_{2}\left(-\frac{9}{16} \sin ^{2} \phi_{1}-\frac{13}{16} \cos ^{2} \phi_{1}\right) \\
& +\frac{1}{16} \varepsilon^{4} \sinh ^{4} 0_{1} \sin ^{2} \phi_{1} \sin ^{2} \theta_{2} .
\end{aligned}
$$

It is convenient to bring the factor $D$ into a form which involves $\phi_{1}$ only in the combination $z_{1}=-\mathrm{i} \sinh 0_{1} \sin \phi_{1}$, because such terms can be expressed as derivatives with respect to $\varepsilon$ of the exponential $\exp \left(\varepsilon z_{1}\right)$ appearing in Eq. (B.15a). This goal can be achieved by integrating by parts all terms that involve $\cos \phi_{1}$. Effectively this amounts to the substitutions $\varepsilon \sinh \theta_{1} \sin \phi_{1} \cos ^{2} \phi_{1} \rightarrow \mathrm{i}\left(\sin ^{2} \phi_{1}-\cos ^{2} \phi_{1}\right)$ and $\varepsilon \sinh \theta_{1} \cos ^{2} \phi_{1} \rightarrow$ i $\sin \phi_{1}$, resulting in

$$
D=\varepsilon z_{1}\left(\frac{7}{2} \sin ^{2} \theta_{2}+2 \sinh ^{2} \theta_{1}\right)+\frac{1}{2} \varepsilon^{2} z_{1}^{2} \sin ^{2} \theta_{2}-\frac{1}{2} \varepsilon^{3} z_{1}^{3} \sin ^{2} \theta_{2} .
$$

Mathematically these expressions are quite similar to those obtained for the decay-rate distribution in Ref. [18]. By a simple substitution rule that relates to each other the terms of different order in $\varepsilon$, we now rewrite $D$ in a way that allows to make direct 
contact to Ref [18], yielding a result in teims of the two functions $\mathscr{T}_{12}$ given in Eq (42) As $1 n$ Ref [18] we express the factors $\left(q_{n}+\lambda_{1}\right)^{-1}$ as an integial of exponential functions

$$
\frac{1}{g_{n}+\lambda_{1}}=\int_{0}^{\infty} \mathrm{d} s_{n} \exp \left[-s_{n}\left(g_{n}+\lambda_{1}\right)\right]
$$

We also write $\left(\lambda_{1}-\lambda_{2}\right)^{2}=\int_{0}^{\infty} \mathrm{d} x x \exp \left[-\lambda\left(\lambda_{1}-\lambda_{2}\right)\right]$ Then the integiations over $0_{1}$ and $\phi_{1}$ can be performed, and $\varepsilon$ only appears in a factor

$$
\Phi\left(\varepsilon, y^{\prime}\right)=\frac{\exp \left[-\sqrt{\varepsilon^{2}+y^{2}}\right]}{\sqrt{\varepsilon^{2}+y^{\prime 2}}},
$$

with $y^{\prime}=y-x-\sum_{n} s_{n}$ The limitıng value for $\varepsilon \rightarrow 0$ of the derivatives

$$
\varepsilon^{n} \frac{\partial^{n}}{\partial \varepsilon^{n}} \Phi\left(\varepsilon, y^{\prime}\right)=C_{n} \delta\left(y^{\prime}\right), \quad C_{1}=-C_{2}=C_{3} / 2=-2
$$

amounts in $\mathrm{Eq}$ (B 16) to the substitutions $\varepsilon^{3} z_{1}^{3} \rightarrow 2 \varepsilon z_{1}$ and $\varepsilon^{2} z_{1}^{2} \rightarrow-\varepsilon z_{1}$, which gives $D=2 \varepsilon z_{1}\left(\lambda_{1}^{2}-\lambda_{2}^{2}\right)$ As a result, we obtain

$$
\begin{aligned}
& {\left[\frac{\Delta}{\pi} P(\Gamma)\right]\langle K\rangle_{\Omega K}=I_{0}(\pi \Gamma / \Delta)+2 I_{1}(\pi \Gamma / \Delta) } \\
& I_{1}(y)=\left.-\frac{1}{4} \lim _{\varepsilon \rightarrow 0} \varepsilon \frac{\partial}{\partial \varepsilon} \int_{1}^{\infty} \mathrm{d} \lambda_{1} \int_{-1}^{1} \mathrm{~d}\right\rangle_{2} \frac{\lambda_{2}^{\prime}}{\left(\lambda_{1}-\lambda_{2}\right)^{\prime}} \\
& \times J_{0}\left(\varepsilon \sqrt{\lambda_{1}^{2}-1}\right) \exp \left[y\left(\lambda_{1}-\lambda_{2}\right)\right] \prod_{n-1}^{N} \frac{g_{n}+\lambda_{2}}{g_{n}+\lambda_{1}},
\end{aligned}
$$

where $J_{0}$ is a Bessel function By compaing expressions with Ref [18], we recognize that $I_{0}(y)=\widetilde{F}_{1}(y) \mathscr{F}_{2}(y)=(\Delta / \pi) P(\Gamma=\Delta y / \pi)$ [cf Eq (42)], while

$$
I_{1}(y)=-\int_{0}^{3} \mathrm{~d} y^{\prime} \mathscr{F}_{1}\left(y^{\prime}\right) \frac{\partial}{\partial y^{\prime}} \mathscr{F}_{2}\left(y^{\prime}\right)
$$

This concludes the deivation of the final iesult $(410)$

\section{References}

[1] A L Schawlow, CH Townes Phys Rev 112 (1958) 1940

[2] K Petemann IEEE J Quant Election 15 (1979) 566

[3] A E Siegman, Phys Rev A 39 (1989) 1253

[4] A E Siegman, Phys Rev A 39 (1989) 1264

[5] Y J Cheng, C G Fanning, A E Siegman, Phys Rev Lett 77 (1996) 627

[6] M A van Eijkelcnborg, $\AA \mathrm{M}$ Lindberg, M S Thijssen, JP Woerdman Phys Rev Lett 77 (1996) 4314

[7] M A van Eıkelenboig, A M Lindbeig MS Thijssen, J P Woerdman, Phys Rev A 55 (1997) 4556

[8] M Biunel, G Ropats, A Le Floch, F Bietenaker, Phys Rev A 55 (1997) 4563

[9] P Giangreı, J P Poizat Euı Phys J D 1 (1998) 97

[10] A E Sregman, unpublishod

[11] C Mahaux, H A Werdenmullet, Shell-Model Approach to Nucleas Reactions, Noth Holland Amsterdam, 1969 
[12] V A Mandelshtam, H S Taylor, Phys Rev Lett 78 (1997) 3274

[13] F Haakc, Quantum Signatues of Chaos, Springet, Berlın, 1991

[14] M Mehta, Random Matrices, Academıc, New York, 1990

[15] V Sokolov, G Zelevinsky, Phys Lett B 202 (1988) 10

[16] V Sokolov, G Zelevinsky, Nucl Phys A 504 (1989) 562

[17] Y V Fyodorov, H -J Somme1s, Pis'ma Zh Eksp Teo1 F1z 63 (1996) 970 [JETP Lett 63 (1996) 1026]

[18] Y V Fyodorov, H -J Sommess, J Math Phys 38 (1997) 1918

[19] H -J Sommeis, Y V Fyodorov, M Titov, J Phys A 32 (1999) L77

[20] R A Jantk, W Noerenberg, M A Nowak, G Papp, I Zahed, pıcpınt (cond-mal/9902314)

[21] J T Chalkc1, B Mehlig, Phys Rev Lett 81 (1998) 3367

[22] B Mchlig, J T Chalker, prepunt (cond-mat/9906279)

[23] M Patta, H Schomerus, CW J Beenakker, Phys Rev A 61 (2000) 23810

[24] K M Frahm, H Schomerus, M Patıa, C W J Beenakkcr, Euıophys Lett 49 (2000) 48

[25] J J M Verbaarschot, H A Weldenmulle1, M R Z11nbaucr, Phys Rep 129 (1985) 367

[26] J Jeffers, N Imoto, R Loudon, Phys Rev A 47 (1993) 3346

[27] C W J Beenakker, Phys Rev Lett 81 (1998) 1829

[28] P Goldberg, P W Milonn, B Sundaram, Phys Rev A 44 (1991) 1969

[29] C W J Beenakker, Rev Mod Phys 69 (1997) 731

[30] T S Misirpashaev, C W J Bcenakker, Phys Rev A 57 (1998) 2041

[31] F von Oppen, Phys Rev Lett 73 (1994) 798

[32] F von Oppen, Phys Rev E 51 (1995) 2647

[33] Y V Fyodorov, H -J Somme1s, Z Phys B 99 (1995) 123

[34] H A Bethc, Phys Rev 66 (1944) 163

[35] F Haake, F Izraılev, N Lehmann, D Sahe1, H -J Sommeıs, Z Phys B 88 (1992) 359

[36] N Lehmann, D Saher, V V Sokolov, H -J Sommets, Nucl Phys A 582 (1995) 223

[37] A V Andrecv, B D Simons, Phys Rev Lett 75 (1995) 2304

[38] S Kettemann, D Klakow, U Smllansky, J Phys A 30 (1997) 3643

[39] H -J Sommers, A Crisant1, H Sompolinsky, Y Sten, Phys Rev Lett 60 (1988) 1895

[40] K B Efetov, Adv Phys 32 (1983) 53 